\title{
Redundancy gains in simple responses and go/no-go tasks
}

\author{
Matthias Gondan, Christina Götze, and Mark W. Greenlee \\ University of Regensburg, Regensburg, Germany
}

\begin{abstract}
In divided-attention tasks with two classes of target stimuli, participants typically respond more quickly if both targets are presented simultaneously, as compared with single-target presentation (redundant-signals effect). Different explanations exist for this effect, including serial, parallel, and coactivation models of information processing. In two experiments, we investigated redundancy gains in simple and go/no-go responses to auditoryvisual stimuli presented with an onset asynchrony. In Experiment 1, go/no-go discrimination was performed for near-threshold and suprathreshold stimuli. Response times in both the simple and go/no-go responses were well explained by a common coactivation model assuming linear superposition of modality-specific activation. In Experiment 2, the go/no-go task was made more difficult. Participants had to respond to high-frequency tones or right-tilted Gabor patches and to withhold their response for low tones and left-tilted Gabors. Redundancy gains were consistent with coactivation models; however, channel-specific buildup of evidence seems to occur at different speeds in the two tasks. Response times of 1 participant support a serial self-terminating model of modality-specific information processing. Supplemental materials for this article may be downloaded from http://app.psychonomic-journals.org/content/supplemental.
\end{abstract}

Although each sensory system is highly specialized for stimuli of its own modality, everyday perception is usually multisensory (Welch \& Warren, 1986). In order to construct a unified and holistic representation of the environment, the information of the different sensory systems needs to be integrated. Which mechanisms are involved in this integration? One of the most basic experimental setups for investigating multisensory integration mechanisms is the so-called redundant-signals paradigm (Miller, 1982). In such experiments, participants have to respond in the same way to target stimuli of two sources - for example, auditory and visual stimuli (A, V). If both stimuli are presented simultaneously (AV), it is frequently observed that responses are substantially faster for AV than for $\mathrm{A}$ or $\mathrm{V}$ alone. This effect is known as the redundantsignals effect or the redundant-target effect (Diederich \& Colonius, 1991; Miller, 1982, 1986; Schwarz, 1989, 1994; Ulrich \& Miller, 1997).

Several models account for this effect, including serial, parallel, and coactive frameworks of information processing. A well-known parallel model is the separateactivation model, or race model (Miller, 1982). According to this model, the two stimulus components are processed in separate channels, and detection occurs as soon as processing in one of the two channels has finished (parallel processing, first-terminating). This mechanism removes slow channel-specific processing times from the AV distribution (statistical facilitation; Raab, 1962), which results in lower mean response times (RTs) for redundant targets than for single targets. Miller (1982), however, showed that redundancy gains due to statistical facilitation have an upper limit. Assuming that the distributions of the channel-specific processing times are equal for single and redundant targets (context independence; e.g., Luce, 1986, p. 130), the so-called race model inequality states that the proportion of fast responses to redundant stimuli [i.e., cumulative RT distribution (CDF); $F(t)=$ $\mathrm{P}(\mathbf{T} \leq t)]$ never exceeds the sum of the CDFs for single stimuli,

$$
F_{\mathrm{AV}}(t) \leq F_{\mathrm{A}}(t)+F_{\mathrm{V}}(t) .
$$

In a powerful extension of the experimental setup, the two stimulus components are presented with onset asynchrony $\tau \geq 0$ [e.g., $\mathrm{A}(\tau) \mathrm{V}$; i.e., the auditory stimulus is presented $\tau$ milliseconds before the visual stimulus; $\mathrm{V}(\tau) \mathrm{A}$ for the reversed order]. Assuming that the distribution of the component processing times for the two components of $\mathrm{A}(\tau) \mathrm{V}$ is equal over stimulus conditions and over the stimulus onset asynchrony (SOA) range, the race model inequality (Miller, 1986) generalizes to

$$
F_{\mathrm{A}(\tau) \mathrm{V}}(t) \leq F_{\mathrm{A}}(t)+F_{\mathrm{V}}(t-\tau),
$$

with $\tau=0$ denoting the special case of synchronous stimulus presentation. In simple response tasks, participants have to press a single response button as soon as they detect an auditory or a visual signal. Here, the upper bound given by Inequality 1 has often been shown to be violated (e.g., Miller, 1982, 1986), and the parallel first-

M.W. Greenlee, mark.greenlee@psychologie.uni-regensburg.de 
terminating processing has been ruled out as the explanation for the redundancy gains observed.

Coactivation models assume that the information of the two channels is integrated at a specific processing stagefor example, by linear superposition of channel-specific activation (Diederich, 1995; Schwarz, 1989, 1994). The diffusion superposition model (Schwarz, 1994) assumes that upon presentation of a stimulus, sensory evidence is accumulated over time. At time $t=0$, the process starts at $\mathbf{X}(0)=0$. Accumulation of evidence is conceived as a noisy diffusion process $\mathbf{X}(t)$ with drift $\mu$ and variance $\sigma^{2}>0$. In each time unit, $\mathbf{X}(t)$ is increased by a normally distributed random increment with mean $\mu$ and variance $\sigma^{2}$. Detection occurs when an evidence criterion $c>0$ is met for the first time. The processing time corresponds to the first passage time $\mathbf{D}$ of the evidence criterion, which, for $\mu>0$, is described by the inverse Gaussian distribution with mean $\mathrm{E}[\mathbf{D}]=c / \mu$.

In redundant targets, coactivation occurs: Here, the diffusion superposition model assumes that the two channel-specific processes superimpose linearly-that is, $\mathbf{X}_{\mathrm{AV}}(t)=\mathbf{X}_{\mathrm{A}}(t)+\mathbf{X}_{\mathrm{V}}(t)$. The new process $\mathbf{X}_{\mathrm{AV}}(t)$ is again a diffusion process with drift $\mu_{\mathrm{AV}}=\mu_{\mathrm{A}}+\mu_{\mathrm{V}}$ and variance $\sigma_{\mathrm{AV}}^{2}=\sigma_{\mathrm{A}}^{2}+\sigma_{\mathrm{V}}^{2}$ (the covariance term is assumed to be zero in Schwarz, 1994). Since the drift parameters add up, $\mathbf{X}_{\mathrm{AV}}(t)$ reaches the criterion earlier than any of its constituents, resulting in faster mean responses to redundant stimuli, $\mathrm{E}\left[\mathbf{D}_{\mathrm{AV}}\right]=c /\left(\mu_{\mathrm{A}}+\mu_{\mathrm{V}}\right)$. What happens with asynchronous stimuli-for example, V67A? During the first $67 \mathrm{msec}$, sensory evidence is accumulated solely by the visual channel. If the criterion $c$ is traversed during this interval, the stimulus is detected, and a response is initiated. Otherwise, the process has attained a subthreshold activation level $x<c$. Starting at $67 \mathrm{msec}$, both channels contribute to the buildup of activity, resulting again in an aggregate process drifting with $\mu_{\mathrm{A}}+\mu_{\mathrm{V}}$ toward a residual barrier $c-x$. Analytic solutions for the mean and the variance of the processing time for asynchronous stimuli have been derived by Schwarz (1994, Equation 10 and Appendix B).

In order to derive a prediction for the observed RTs $\mathbf{T}$, an additional variable $\mathbf{M}$ has to be introduced. This variable summarizes residual processes not described by the model (e.g., peripheral perceptual processes, response preparation, motor execution, etc.), so that $\mathbf{T}=\mathbf{D}+\mathbf{M}$ (e.g., Luce, 1986, chap. 3). Schwarz (1994) demonstrated that superposition of diffusion processes accurately predicts the means and standard deviations for simple manual responses to auditory-visual stimuli. The function describing the relationship between SOA and mean RT has a wing shape, with a minimum at simultaneous stimulus presentations and asymptotic behavior at higher onset asynchronies (e.g., Schwarz, 1994, Figure 1). In Appendix A, we show that the diffusion superposition model predicts a violation of the race model inequality.

Auditory-visual redundancy gains have been studied most extensively using simple response tasks (e.g., Miller, 1982, Experiments 1 and 2; Miller, 1986; Raab, 1962; Schwarz, 2006). In the simple response task (Type A re- sponse; Donders, 1868/1969), observers have to press a button upon appearance of any stimulus. In more complex tasks - for example, go/no-go tasks - participants do not have to respond to all stimuli but are asked to discriminate a target (or a set of targets) among (a set of) distractor stimuli (Type C response; Donders, 1868/1969). Redundancy gains have been observed in go/no-go tasks (Gondan, Niederhaus, Rösler, \& Röder, 2005; Grice \& Canham, 1990; Grice \& Reed, 1992, Experiments 1 and 2; Miller, 1982, Experiment 3; Teder-Sälejärvi, McDonald, Di Russo, \& Hillyard, 2002) and choice responses (Grice, Canham, \& Boroughs, 1984; Grice, Canham, \& Gwynne, 1984). The aim of the present study was to investigate whether redundancy gains observed in different tasks (simple responses, go/no-go responses) are based on the same mechanismthat is, linear superposition of modality-specific activity.

For this purpose, we tested whether the diffusion superposition model (Schwarz, 1994) can be adjusted to simple responses and to go/no-go responses to auditoryvisual stimuli presented with different SOAs. In Experiment 1 , target stimuli had high intensity, and distractor stimuli had low intensity. Separate diffusion superposition models were adjusted to the two tasks, and a common model describing behavior in both tasks was tested. We hypothesized that in Experiment 1, participants would easily distinguish between targets and distractors. As compared with the simple response task, we hypothesized that an increased evidence barrier $c$ would be observed in the go/no-go task. Different task requirements were modeled by a different residual M. In Experiment 2, targets and distractors had the same intensity. Participants had to discriminate high- and low-frequency tones and leftand right-tilted Gabor patches. The go/no-go task was, thus, more difficult, raising the question of whether redundancy gains can be explained by simple superposition of neural activity. Here, we additionally tested whether the observed RTs would support a serial self-terminating model of information processing (e.g., Townsend \& Ashby, 1983).

\section{EXPERIMENT 1}

Auditory and visual stimuli of two intensities (a, A, v, V, with lowercase letters representing low-intensity stimuli and uppercase letters representing high-intensity stimuli) were delivered either alone or in pairs with different onset asynchronies. In the simple response task, participants had to respond to any stimulus by pressing, as quickly as possible, a single button. In the go/no-go task, participants were again instructed to respond as quickly as possible, but only if a stimulus with high intensity was delivered in one of the two modalities.

\section{Method}

Participants. Three participants took part in the study ( 1 of them male, 2 female; 33,22 , and 45 years old, respectively). All the participants were students at the University of Regensburg. For their participation, they received either course credit or $€ 7$ per hour. Every participant was tested during four sessions, each lasting approximately $3 \mathrm{~h}$, until 21 blocks for both tasks were recorded. 
Stimuli and experimental conditions. The experiments took place in a dimly lit, sound-attenuated room. Visual stimuli were two Gabor patches ( $1^{\circ}$ diameter, 4 cycles $/ \mathrm{deg}$, tilted $30^{\circ}$ to the right), presented on a 21-in. CRT monitor (background luminance, $16 \mathrm{~cd} / \mathrm{m}^{2}$ ), simultaneously $5^{\circ}$ to the left and to the right side of a fixation spot. Lateralized stimulus presentation was necessary because, in a pilot study, weak central stimuli turned out to be so salient that the mean RTs for centrally presented weak and strong visual stimuli did not significantly differ. Moreover, presenting visual and auditory stimuli left and right of central fixation allows for manipulation of the distance between the stimulus components (e.g., Gondan et al., 2005; Schwarz, 2006) in follow-up experiments. Visual stimuli were either of high intensity (V; Michelson contrast, .98) or of low intensity (v; Michelson contrast, .20). Auditory stimuli were noise bursts of high intensity (A, $45 \mathrm{dBA}$ ) or low intensity (a, $29 \mathrm{dBA}$ ), delivered via two loudspeakers placed left and right of the screen. Each stimulus was presented for a maximum of $1,000 \mathrm{msec}$, or it was terminated by the response of the participant. The auditory and visual stimuli were presented either alone (unimodal condition; a, v, A, V) or together (bimodal conditions; av, Av, aV, AV), with onset asynchronies of $0 \mathrm{msec}$ (synchronous condition) or $\pm 33, \pm 67, \pm 100, \pm 133$, or $\pm 167 \mathrm{msec}$ (asynchronous conditions), negative values indicating that the auditory stimulus preceded the visual stimulus. The distance between the chinrest and the computer monitor and the speakers was $70 \mathrm{~cm}$. The participants responded to the targets by pushing the left button of a response box with the index finger of their right hands.

Procedure. For the simple response task, the participants were instructed to respond as quickly as possible to any stimulus delivered. During the go/no-go blocks, they were instructed to respond as quickly as possible to stimuli containing a high-intensity component (A, V, AV, Av, aV) and to suppress a response if only low-intensity stimuli were presented (a, $v, a v)$.

Simple response blocks and go/no-go blocks were run in alternating order. After 5 blocks, there was a break (break duration was determined by the participant but was at least $5 \mathrm{~min}$ ) in order to prevent fatigue and loss of motivation, and additional breaks were taken if deemed necessary by the participant. In each block, 5 stimuli of each of the 48 SOA $\times$ intensity conditions plus 5 catch trials were randomly intermixed, with the intertrial interval varying uniformly between 1,700 and 2,300 msec. In each simple response block, the participants had to respond to 240 stimuli. In each go/no-go block, the participants had to respond to 175 stimuli and suppress a response to 65 stimuli. Within each block, the stimuli were presented in randomized order. The participants completed 21 blocks of simple responses and 21 blocks of go/no-go responses, each block lasting about $14 \mathrm{~min}$. This took approximately $12 \mathrm{~h}$ per participant, with maximal session duration of $3 \mathrm{~h}$. Altogether, 5,040 responses were collected in the simple response task and 3,675 responses in the go/ no-go task.

Test of the race model inequality. For the simple response task, the race model was tested for all possible stimulus combinations ( $\mathrm{AV}, \mathrm{Av}, \mathrm{aV}$, av). For the go/no-go task, the race model inequality was tested only for condition AV, where both stimulus components were targets.

Because the race model inequality (1) is asymmetric, with two summands on the right side and only one term on the left side, fast guesses inflate the right side of the race inequality so that the race model might erroneously be accepted (Miller \& Lopes, 1991). To avoid such a distortion of the results, catch trials (i.e., trials without stimulation) can be used to apply the so-called kill-the-twin correction (Eriksen, 1988). In the kill-the-twin procedure, the distribution of fast guesses is estimated from erroneous responses to catch trials. For each response to a catch trial, one correct response of similar speed is eliminated from the A, V, and AV distributions. This is equivalent to the subtraction of the RT distribution for the catch trials from each term of Inequality 1 :

$$
\begin{aligned}
{\left[F_{\mathrm{A}(\tau) \mathrm{V}}(t)-F_{\mathrm{C}}(t)\right] } & \leq\left[F_{\mathrm{A}}(t)-F_{\mathrm{C}}(t)\right] \\
& +\left[F_{\mathrm{V}}(t-\tau)-F_{\mathrm{C}}(t-\tau)\right] .
\end{aligned}
$$

Because $F_{C}(t)$ cancels out on the two sides of Inequality 2, only the RT distribution of the second stimulus needs to be corrected (for more details and a justification of the procedure, see Gondan \& Heckel, 2008).

For the go/no-go task, fast guesses were corrected on the basis of erroneous responses to distractor stimuli: For each erroneous response observed to an auditory distractor (a), a similar response was eliminated from the responses to auditory target stimuli (A). For each response to $\mathrm{v}$, a similar response was eliminated from the responses to $\mathrm{V}$, and so on, for all SOAs:

$$
\begin{aligned}
{\left[F_{\mathrm{A}(\tau) \mathrm{V}}(t)-F_{\mathrm{a}(\tau) \mathrm{v}}(t)\right] } & \leq\left[F_{\mathrm{A}}(t)-F_{\mathrm{a}}(t)\right] \\
& +\left[F_{\mathrm{V}}(t-\tau)-F_{\mathrm{v}}(t-\tau)\right] .
\end{aligned}
$$

Using the CDFs for each target condition, the observed violation area $\Delta$ was calculated for each participant and condition. This is the area below the CDF of the redundant stimulus (left-hand side of Inequalities 2 and 3) but above the sum of the CDFs of the unimodal stimuli (right-hand side of Inequalities 2 and 3). If the race model holds, $\Delta=0$. To rule out an accidental violation of the race model due to sampling error, RTs for each experimental condition for each participant were simulated by the computer assuming a race model. Simulated RTs for unimodal stimuli were obtained by bootstrapping from the observed distribution for the unimodal RTs for each participant, after correcting for fast guesses. Simulated RTs for bimodal stimuli were obtained by sampling pairs of RTs from the observed distributions for unimodal stimuli, adjusting them for SOA, and by choosing the smaller of the two values. Note that sampling was not independent but that fast auditory RTs were combined with slow visual RTs (for details and a justification of this procedure, see Miller, 1986, pp. 336-337). This simulation was carried out 10,000 times, resulting in 10,000 violation areas $\Delta^{*}$ simulated under the null hypothesis (race model). Comparable to a standard one-sided hypothesis test, the race model is rejected if the observed $\Delta$ is greater than $95 \%$ of the simulated $\Delta^{*}$.

Performing one race model test per SOA leads to an increase of the Type I and Type II errors. This problem was addressed by weighting the SOA-specific violation areas $\Delta_{\tau}$ with $\lambda(\tau)>0$ and adding them up to an overall violation area (Gondan, 2009):

$$
\Delta_{\text {total }}=\Sigma_{\tau} \lambda(\tau) \times \Delta_{\tau} .
$$

Under the race model, $\Delta_{\tau}=0$ for all SOAs. Thus, the weighted sum of all $\Delta_{T} \mathrm{~S}$ should equal zero as well. In simple responses to auditoryvisual redundant stimuli, Miller (1986) noted that coactivation effects were highest with SOAs that roughly equalized the mean RTs to unimodal stimuli. Therefore, the weighting function $\lambda(\tau)$ was chosen to emphasize the SOAs in which coactivation effects were expected to be high. We used a triangular function maximal around the difference between the mean RTs observed for single visual and auditory stimuli $\left(M_{\mathrm{V}}-M_{\mathrm{A}}\right)$.

Model adjustment and goodness of fit. For each SOA and condition ( $\mathrm{av}, \mathrm{AV}, \mathrm{aV}, \mathrm{Av}$ ), 100 responses were collected, resulting in 4,800 simple responses and 3,500 go/no-go responses. To avoid a distortion of the results due to unusually fast or slow responses, RTs outside the percentile range $[0.5 \%, 99.5 \%]$ were discarded. These percentiles were calculated on the basis of the entire participantspecific RT distribution, collapsed across conditions and SOAs. Corresponding to the two intensity levels for each modality, parameters of the diffusion processes were distinguished into low- and high-intensity parameters: $\mu_{\mathrm{V}}$ and $\sigma_{\mathrm{V}}^{2}$ (high intensity) and $\mu_{\mathrm{V}}$ and $\sigma_{\mathrm{V}}^{2}$ (low intensity) for the visual process; $\mu_{\mathrm{A}}$ and $\sigma_{\mathrm{A}}^{2}$, as well as $\mu_{\mathrm{a}}$ and $\sigma_{\mathrm{a}}^{2}$, for the auditory process. The absorbing barrier (criterion) was set to $c=100$, since it only scales the other parameters (Schwarz, 1994). Drifts and variances of the auditory diffusion processes $\left(\mu_{\mathrm{a}}, \mu_{\mathrm{A}}, \sigma_{\mathrm{a}}^{2}, \sigma_{\mathrm{A}}^{2}\right)$ and those of the visual diffusion processes $\left(\mu_{\mathrm{v}}, \mu_{\mathrm{V}}\right.$, $\left.\sigma_{\mathrm{V}}^{2}, \sigma_{\mathrm{V}}^{2}\right)$, as well as the expected value for the duration of residual processes $\mu_{\mathrm{M}}$, were iteratively adjusted to the observed mean RTs by minimizing a goodness-of-fit $\chi^{2}$ value (see below). Technically, this minimization was performed using the constrOptim command 
of the R statistical language (R Development Core Team, 2009) with constraints $0.1 \leq \mu_{\mathrm{a}}, \mu_{\mathrm{A}}, \mu_{\mathrm{v}}, \mu_{\mathrm{V}} \leq 10$ for the drifts, $0.1 \leq \sigma_{\mathrm{a}}^{2}, \sigma_{\mathrm{A}}^{2}$, $\sigma_{\mathrm{V}}^{2}, \sigma_{\mathrm{V}}^{2} \leq 100,000$ for the variances, and $100 \leq \mu_{\mathrm{M}} \leq 500$ for the mean residual. In the go/no-go task, negative drifts were allowed for no-go components; constraints were $-1 \leq \mu_{\mathrm{a}}, \mu_{\mathrm{v}} \leq 10$.

The observed mean RTs are based on sums of a large number of independent replications ( $N=100$ responses per SOA, possibly less due to trimming and omitted responses) and can thus be assumed to follow a normal distribution due to the central limit theorem. First, for each condition, standardized means were calculated by subtracting the predicted mean $\mathrm{E}[\mathrm{T}]$ from the observed mean $M$ and dividing the result by the observed standard error $\hat{s} / \sqrt{ } N$. Thereby, for each condition, a $t$ value with $N-1$ degrees of freedom $(d f \mathrm{~s})$ was obtained. Due to the high number of $d f \mathrm{~s}$, this standardized mean approximately followed a normal distribution,

$$
z_{\mathrm{A}(\tau) \mathrm{V}}=\left\{M_{\mathrm{A}(\tau) \mathrm{V}}-\mathrm{E}\left[\mathbf{T}_{\mathrm{A}(\tau) \mathrm{V}}\right]\right\} /\left\{\hat{s}_{\mathrm{A}(\tau) \mathrm{V}} / \sqrt{ } N_{\mathrm{A}(\tau) \mathrm{V}}\right\} \sim N(0,1),
$$

with a mean of zero if the model holds. Stated differently, if the observed mean RTs are in accordance with the predictions of Schwarz's (1994) model, $z_{\mathrm{A}(\tau) \mathrm{V}}$ should lie within \pm 1.96 in $95 \%$ of the cases.

By squaring the $z$ values for each SOA and intensity condition, $48 \chi^{2}(1)$ values were obtained for the simple response task. Since the model has nine free parameters that are adjusted to the mean RTs, the sum of these $\chi^{2}$ values has $39 d$ fs (e.g., Bulmer, 1979, p. 156f; Good, 1973):

$$
\Sigma_{\tau} z_{\mathrm{A}(\tau) \mathrm{V}^{2} \sim \chi^{2}(39)}
$$

Model adjustment to the go/no-go responses was performed in the same way. Because, in the go/no-go task, participants had to withhold their response to av stimuli, mean RTs for only 35 conditions were available ( $\mathrm{AV}, \mathrm{Av}, \mathrm{aV})$. The barrier $c$ was fixed at the value determined by the fit of the common model for simple and go/no-go responses (see below).

In a third model fit, the diffusion superposition model was simultaneously adjusted to the mean RTs for the simple response task and the go/no-go task. For this common model, we used only RTs for high-intensity stimuli (A, V, AV). The physical properties and the meaning of these stimuli were identical in the two tasks; thus, the diffusion parameters $\mu_{\mathrm{A}}, \sigma_{\mathrm{A}}^{2}, \mu_{\mathrm{V}}, \sigma_{\mathrm{V}}^{2}$ were assumed to be equal for simple and go/no-go responses. The different requirements of the two tasks were accounted for by enabling different evidence criteria $c$ and different mean residual times $\mu_{\mathrm{M}}$. Again, a goodness-of-fit $\chi^{2}$ value was calculated using the observed and predicted mean RTs for the 13 SOAs in the two tasks, resulting in 26 predictions of an aggregate model with seven parameters. Therefore, the $\chi^{2}$ value of the combined model of simple responses and go/no-go responses has $26-7=19 d f$ s for each participant.

The adjusted models describe only the mean RTs (see, e.g., Schwarz, 2006), although Schwarz (1994, Appendix B) derived an explicit prediction for the variance of $\mathbf{D}$ for different SOAs. Using two additional parameters for the variance of $\mathbf{M}$ and the correlation between $\mathbf{D}$ and $\mathbf{M}\left(\sigma_{\mathrm{M}}^{2}, \rho_{\mathrm{DM}}\right)$, it is possible to describe the variance of the observed RTs as well. The simultaneous fit of the observed mean RTs and variances is, however, substantially more complex. Most important, the observed mean RTs and variances are not independent for a given experimental condition (sample mean and variance are independent only for normally distributed variables), which would be necessary to sum up the observed squared standardized discrepancies as in the goodness-of-fit statistic (Equation 6). Consequently, we tested the model only for the observed mean RTs, for which goodness-of-fit statistics could be determined using standard $\chi^{2}$ techniques.

\section{Results}

Mean RTs per condition. Table S1 (supplemental materials) shows the mean RTs for the simple response task, separately for each participant, SOA, and intensity.
The relationship between SOA and mean RT exhibited a wing shape, with fastest responses in the synchronous AV condition (Figure 1, left panels). In 2 of the 3 participants, the number of omitted responses was unexpectedly high for the unimodal, low-intensity stimuli, indicating that these participants had difficulties in perceiving the signals. Whereas Participant 1 anticipated about $25 \%$ of the trials, the other 2 participants showed more conservative response behavior. Mean RTs slightly increased within experimental sessions $(1.8,0.9$, and $2.6 \mathrm{msec} / \mathrm{block}$ for Participants 1, 2, and 3, respectively).

Mean RTs observed in the go/no-go task were substantially higher than those in the simple response task (supplemental Table S2, Figure 1, middle panels). In the no-go condition (both stimuli of low intensity, av), the number of false alarms was substantial, indicating that the participants used a liberal criterion for responding. Again, we noted a slight RT increase during the experimental sessions, as reflected by a positive regression slope of block number and mean RT $(0.3,4.9$, and $0.5 \mathrm{msec} / \mathrm{block}$ for the 3 participants).

Test of the race model. For the simple response task, the race model inequality was tested for all intensity conditions (AV, Av, aV, av). The weighted sum of SOAspecific violation areas is shown in Figure 2. The $p$ values for these aggregate violation areas under the race model assumption are shown in Table 1 for each participant. In each participant, significant violations of the race model inequality were observed for at least one intensity combination (AV, Av, aV, av).

No responses to unimodal low-intensity stimuli were available for the go/no-go task. Therefore, the race model was tested only for condition AV (Table 1, Figure 2). A significant violation of the race model prediction was found for Participant 3; for the other 2 participants, RT distributions did not rule out race models for the redundancy gains observed.

Diffusion superposition model. The diffusion superposition model predicts the unconditional mean RTs for a specific condition $(\mathrm{E}[\mathrm{T}])$; it does not predict the mean RTs conditional on detection within a specific interval (e.g., $\mathrm{E}[\mathbf{T} \mid \mathbf{T}<1,000])$. Due to the high number of omitted responses to weak unimodal stimuli (a, v; see Table S1), mean RTs observed for these two conditions had to be excluded from the analysis. This affects only the simple response task, in which the number of observed means was 46 instead of 48 . Drifts and variances of the auditory $\left(\mu_{\mathrm{a}}, \mu_{\mathrm{A}}, \sigma_{\mathrm{a}}^{2}, \sigma_{\mathrm{A}}^{2}\right)$ and the visual $\left(\mu_{\mathrm{V}}, \mu_{\mathrm{V}}, \sigma_{\mathrm{V}}^{2}, \sigma_{\mathrm{V}}^{2}\right)$ diffusion processes, as well as the mean of the residual component $\mu_{\mathrm{M}}$, were adjusted to the mean RTs recorded in the simple response task. The parameters of the fitted model are listed in Table 2 (left column).

Corresponding to the higher mean RTs to low-intensity stimuli, drifts and variances for low-intensity stimuli were smaller than drifts and variances for high-intensity stimuli. The diffusion variance parameters $\sigma_{\mathrm{a}}^{2}, \sigma_{\mathrm{A}}^{2}, \sigma_{\mathrm{v}}^{2}, \sigma_{\mathrm{V}}^{2}$ seemed to be roughly proportional to the drift parameters (Tolhurst, Movshon, \& Dean, 1983). The mean RTs observed in the simple response task were in good agreement with 
Simple Responses (Participant 1)

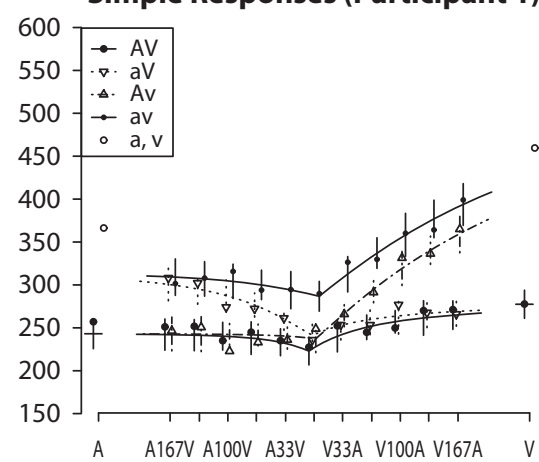

Participant 2

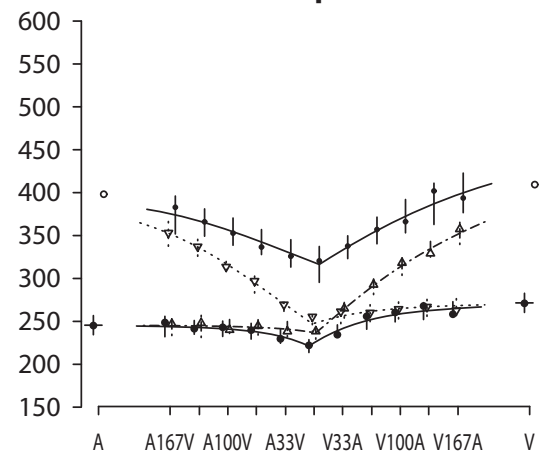

Participant 3

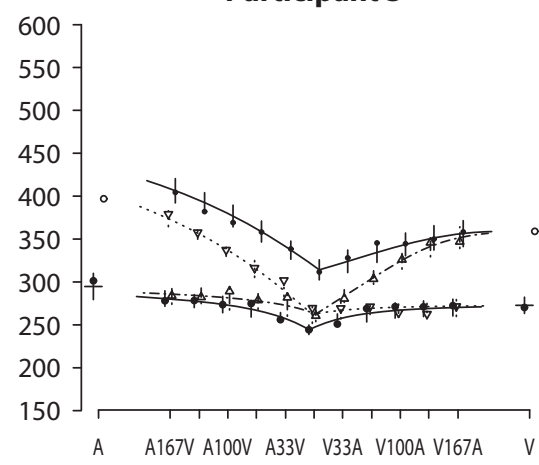

Go/No-Go (Participant 1)

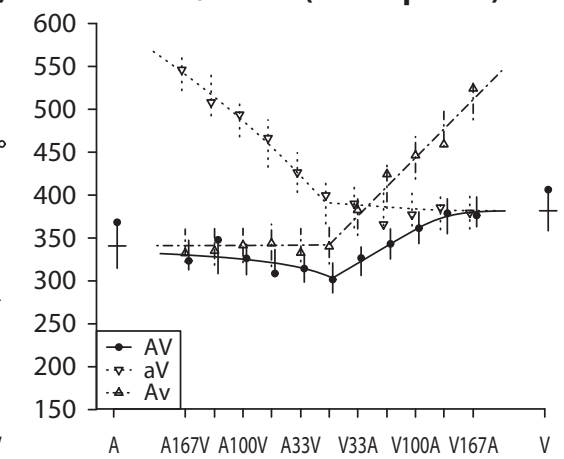

Participant 2

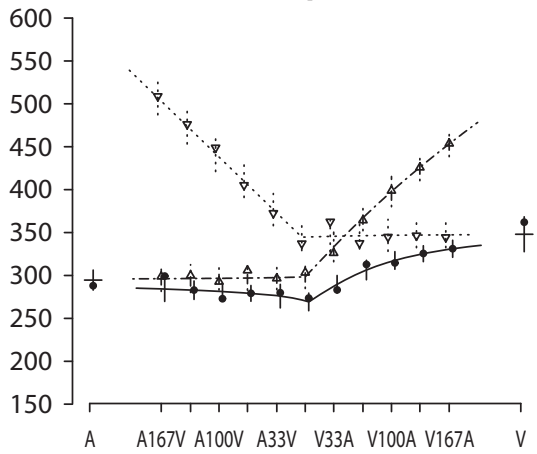

Participant 3

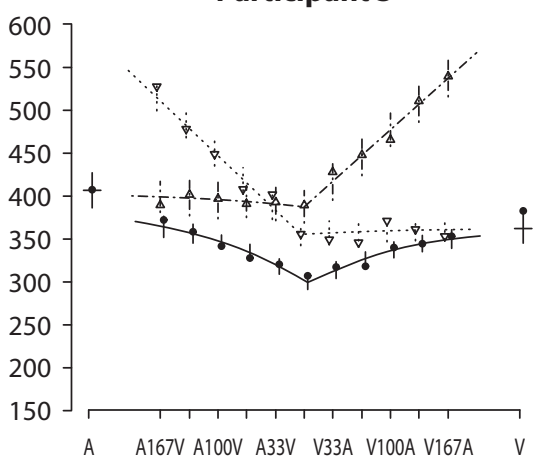

Common Fit (Participant 1)

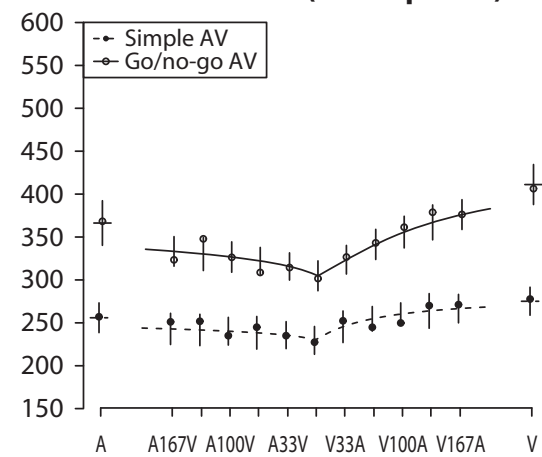

Participant 2

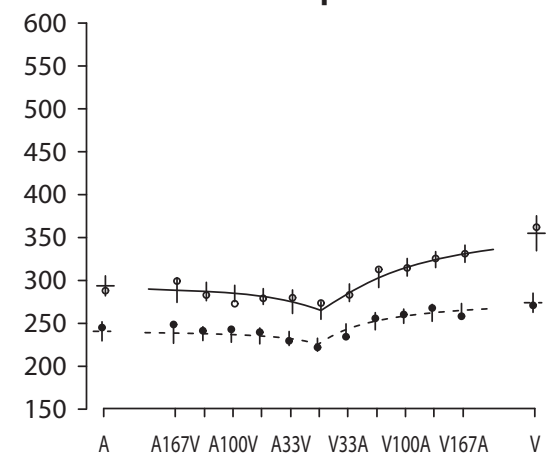

Participant 3

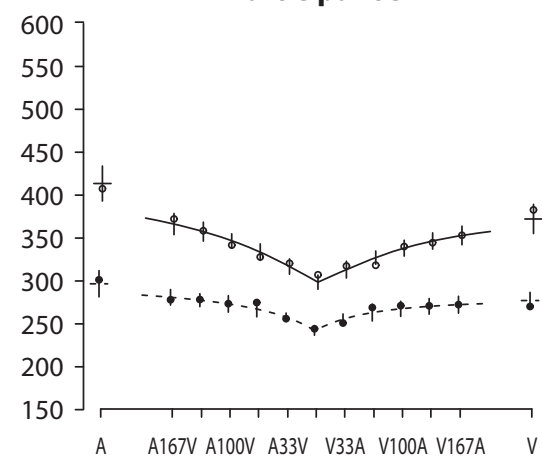

Figure 1. Left panels: Diffusion superposition model for simple responses to auditory-visual stimuli of different onset asynchrony and intensity ( $\mathrm{AV}, \mathrm{Av}, \mathrm{aV}$, av) in Experiment 1. Wing-shaped line: Model prediction of mean response times, including $95 \%$ confidence intervals. The confidence intervals were calculated assuming normally distributed means, with standard errors based on the observed standard deviations. Symbols: Observed mean response times for the different conditions. Unimodal, weak intensity stimuli (a, v) were not used for model adjustment and test. Center panels: Same for go/no-go task. Right panels: Combined diffusion superposition model of simple responses (conditions $\mathrm{A}, \mathrm{V}, \mathrm{AV}$ only) and go/no-go responses (conditions $\mathrm{A}, \mathrm{V}, \mathrm{AV}$ only).

the prediction of the fitted model [overall goodness of fit, $\left.\chi^{2}(111)=111.68, p=.464\right]$. Figure 1 (left panels) illustrates the relationship between SOA and mean RT for the different intensity combinations.

In a second model fit, the diffusion superposition model was adjusted to the mean RTs of go/no-go conditions AV, $\mathrm{Av}$, and $\mathrm{aV}$. To enable a qualitative comparison of the drift rates estimated for the two tasks, the criterion $c$ was fixed at the value resulting from the combined fit of the simple response and the go/no-go task (see the next paragraph). Table 2 (middle columns) shows the parameter estimates for the 3 participants. Drift rates for the high-intensity target components were comparable to those for the simple response task. Drift rates for the low-intensity nontarget components were very small (and sometimes negative), indicating that the nontarget components had virtually no effect on the observed mean RTs. Model fit was acceptable $\left[\chi^{2}(78)=99.0, p=.054\right]$, indicating that the superposition model adequately describes the mean RTs observed in the go/no-go task as well.

In the last step, the diffusion model was simultaneously adjusted to the mean RTs for the high-intensity AV stimuli 
Race Model Test (Participant 1)
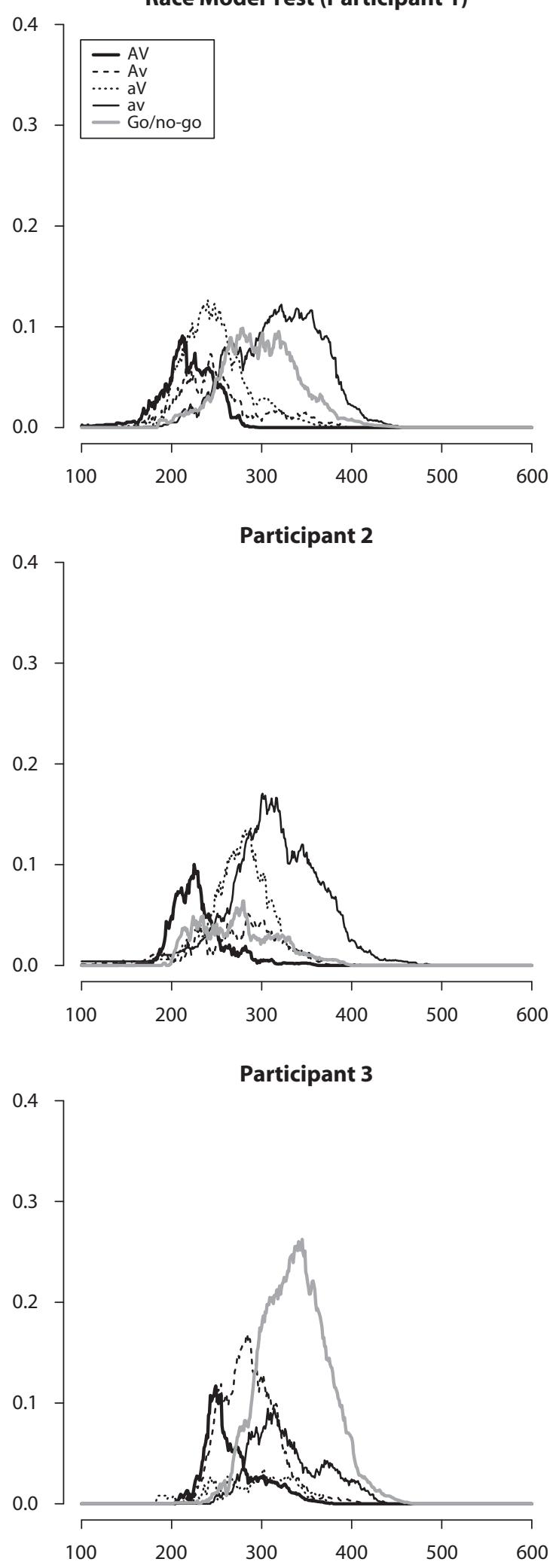

Figure 2. Experiment 1: Test of the race model inequality. Weighted sum of SOA-specific violation areas (Equation 4) for the different conditions of the simple task ( $\mathrm{AV}, \mathrm{Av}, \mathrm{aV}$, av) and the go/no-go task (only AV condition). For significance tests on these violation areas, see Table 1.
Table 1

Experiment 1: Race Model Test ( $p$ Values)

\begin{tabular}{ccccc}
\hline & \multicolumn{3}{c}{ Participant } & \\
\cline { 2 - 4 } Task & 1 & 2 & 3 & Summary \\
\hline Simple & & & & \\
AV & .245 & .068 & .021 & .014 \\
aV & .264 & .010 & .206 & .020 \\
aV & .037 & .006 & .643 & .007 \\
Av & .255 & .045 & .004 & .003 \\
Go/no-go & & & & \\
AV & .108 & .134 & $<.001$ & .001 \\
\hline
\end{tabular}

Note-The Fisher omnibus test was used for the summary statistic: $-2 \sum \ln P_{i} \sim \chi^{2}(2 N)$.

of the two tasks. Since the physical properties and the response relevance of these AV stimuli were identical in the two tasks, diffusion parameters describing the detection process $\mathbf{D}$ were assumed to be equal in both tasks. The different requirements of the two tasks were accounted for by allowing a variable evidence criterion $c$ and different residual components $\mu_{\mathrm{M}}$ in the two tasks. The goodness of fit of this aggregate model was still acceptable $\left[\chi^{2}(57)=\right.$ $79.7, p=.025]$, indicating that the combined diffusion superposition model can describe behavior in both tasks. Figure 1 (right panels) illustrates the good agreement of model prediction and observed data. The adjusted parameters are shown in Table 2 (right column). In the go/no-go task, $c$ was elevated for all the participants, indicating that the participants used a higher evidence criterion for distinguishing go and no-go trials. The residual component $\mathbf{M}$ had a slightly higher mean in the go/no-go task as well.

\section{Discussion}

In bimodal divided attention, redundant-signal effects have been extensively investigated using simple manual RT tasks (e.g., Diederich \& Colonius, 1987, 1991; Miller, 1982, 1986; Miller \& Ulrich, 2003; Schwarz, $1989,1994)$. A common finding has been that parallel race models cannot explain the redundancy gains, and coactivation models have been developed that are well supported by the observed RTs (Diederich, 1995; Miller, 1982, Appendix; Miller \& Ulrich, 2003; Schwarz, 1989, 1994). In go/no-go tasks, redundant-signals effects similar to those in simple RT have frequently been reported (Gondan et al., 2005; Grice \& Canham, 1990; Grice \& Reed, 1992; Miller, 1982, Experiment 3; Teder-Sälejärvi et al., 2002). In most studies with auditory-visual stimulus arrangements, the upper bound for the redundancy gain defined by Inequality 1 was consistently violated, ruling out the parallel race model from consideration. The results obtained in Experiment 1 replicate this observation, although violations of the race model prediction were weak and were statistically significant for only 1 participant (Figure 2, Table 1).

The working hypothesis of Experiment 1 is that linear superposition of channel-specific activation is responsible for the redundancy gains observed in both tasks. This hypothesis was tested by fitting the diffusion superposition model (Schwarz, 1994) to the mean RTs observed in the simple response task and in the go/no-go task. In the 
Table 2

Experiment 1: Superposition Model for Simple Responses and Go/No-Go Responses and Common Model for Both Tasks-Parameter Values and Goodness of Fit (GOF)

\begin{tabular}{|c|c|c|c|c|c|c|c|c|c|}
\hline & \multicolumn{3}{|c|}{ Simple } & \multicolumn{3}{|c|}{ Go/No-Go } & \multicolumn{3}{|c|}{ Simple and Go/No-Go } \\
\hline & $\begin{array}{c}\text { Participant } \\
1\end{array}$ & $\begin{array}{c}\text { Participant } \\
2\end{array}$ & $\begin{array}{c}\text { Participant } \\
3\end{array}$ & $\begin{array}{c}\text { Participant } \\
1\end{array}$ & $\begin{array}{c}\text { Participant } \\
2\end{array}$ & $\begin{array}{c}\text { Participant } \\
3\end{array}$ & $\begin{array}{c}\text { Participant } \\
1\end{array}$ & $\begin{array}{c}\text { Participant } \\
2\end{array}$ & $\begin{array}{c}\text { Participant } \\
3\end{array}$ \\
\hline$\mu_{\mathrm{v}}$ & 0.23 & 0.30 & 0.65 & -0.04 & -0.14 & 0.13 & - & - & - \\
\hline$\sigma_{\mathrm{v}}^{2}$ & 48.0 & 41.9 & 6.5 & 12.0 & 189.7 & 8.9 & - & - & - \\
\hline$\mu_{\mathrm{V}}$ & 1.14 & 1.17 & 1.51 & 1.76 & 1.49 & 1.46 & 1.25 & 1.31 & 1.28 \\
\hline$\sigma_{V}^{2}$ & 205.5 & 109.8 & 157.9 & 18.7 & 224.8 & 141.8 & 194.4 & 212.9 & 146.8 \\
\hline$\mu_{\mathrm{a}}$ & 0.81 & 0.47 & 0.28 & -0.12 & 0.04 & 0.07 & - & - & - \\
\hline$\sigma_{\mathrm{a}}^{2}$ & 52.0 & 13.5 & 52.7 & 345.4 & 7.4 & 8.2 & - & - & - \\
\hline$\mu_{\mathrm{A}}$ & 1.88 & 1.68 & 1.13 & 2.55 & 2.62 & 1.12 & 1.64 & 2.31 & 1.02 \\
\hline$\sigma_{\mathrm{A}}^{2}$ & 170.5 & 159.3 & 268.8 & $1,398.5$ & $3,373.6$ & 276.8 & $2,183.2$ & 600.1 & 220.9 \\
\hline$c_{\mathrm{S}}$ & $(100)$ & $(100)$ & (100) & - & - & - & $(100)$ & $(100)$ & $(100)$ \\
\hline$\mu_{\mathrm{M}, \mathrm{S}}$ & 189.8 & 186.2 & 206.5 & - & - & - & 194.8 & 197.5 & 198.9 \\
\hline$c_{\mathrm{G}}$ & - & - & - & (233.1) & $(183.8)$ & $(211.5)$ & 233.1 & 183.8 & 211.5 \\
\hline$\mu_{\mathrm{M}, \mathrm{G}}$ & - & - & - & 249.3 & 224.3 & 217.5 & 223.9 & 214.2 & 206.6 \\
\hline GOF $\chi^{2}$ & 38.95 & 22.84 & 49.90 & 29.05 & 24.33 & 45.65 & 17.86 & 34.77 & 27.11 \\
\hline$d f$ & 37 & 37 & 37 & 26 & 26 & 26 & 19 & 19 & 19 \\
\hline$p$ & .382 & .967 & $.076^{*}$ & .309 & .557 & .010 & .532 & .015 & . 102 \\
\hline
\end{tabular}

Note-Fixed parameters are given in parentheses. $\quad{ }^{*}$ For Participant 3 , inclusion of condition v (one omission) slightly improves the fit $\left[\chi^{2}(38)=\right.$ $49.92, p=.093]$. If condition a (six omissions) is included, the fit is poor $\left[\chi^{2}(39)=91.45, p<.001\right]$.

simple response task, participants had to respond to all weak and strong stimuli, whereas in the go/no-go task, target stimuli were defined as having at least one component with high intensity. These task-specific model fits were satisfactory (Table 2, Figure 1). In a second step, a single model was fitted to the mean RTs observed in both tasks. For this aggregate model, only high-intensity stimuli (A, V, AV) were used, because these stimulus combinations had the same physical properties and the same response relevance in both tasks. We thus assumed at least a subset of perception-related processes to be identical in the two tasks, and the parameters describing the diffusion processes elicited by the stimulation were forced to be equal in both tasks. Differences between the two tasks were modeled by different evidence barriers and different mean residuals in the two tasks. Consistent with expectation, the barrier $c$ was increased in the go/no-go task (Table 2, right columns), as compared with $c=100$ (fixed) in the simple response task, reflecting an increased criterion for responding in the go/no-go task. Schwarz (1994) noted that even in simple manual responses (Miller, 1986), the residual accounts for a considerable amount of the mean and the variance of the observed RT, which suggests that the residual $\mathbf{M}$ describes much more than just motor execution (e.g., response preparation, peripheral processes). The estimates shown in Table 2 confirm this observation: In line with the increased demands of the go/no-go task, estimates for the residual component were higher for go/ no-go responses than for simple responses.

The goodness of fit ( $p=.025$ ) of this common model is acceptable, but some aspects of the data might not be fully covered: The normal approximation in Equation 5 might be too optimistic, trial-to-trial sequence effects are not described by the model (Gondan, Lange, Rösler, \& Röder, 2004), and the diffusion parameters, as well as the residual $\mathbf{M}$, might not be constant during and across the multiple sessions of the experiment. For example, within sessions, performance decreased over time, as reflected by a positive correlation of the block-specific mean RT and the order of the experimental block within sessions. Given that most of these aspects cause extra variation that works against a good model fit, it seems fair to state that the common diffusion superposition model adequately describes behavior in the two tasks.

As was already mentioned, the common model of simple and go/no-go responses was adjusted without the mean RTs observed in conditions av, Av, aV because the low-intensity components have different relevance in the two tasks: targets in the simple response task but nontargets in the go/no-go task. In line with this, negative drift rates were observed for some of the nontarget components in the go/no-go task. Whereas additive superposition can be hypothesized to account for the redundancy gain observed in the simple response task, nontarget components lengthen the mean RT observed in go/no-go responses to combinations of targets and nontargets (cf. the so-called diffusion difference model; Schwarz, 2006).

\section{EXPERIMENT 2}

In Experiment 2, participants again made simple and go/no-go responses to auditory-visual stimuli presented at different SOAs. In Experiment 2, go and no-go stimuli had the same intensity but differed in orientation (Gabor stimuli) and tone frequency (low, high). We expected that this discrimination task would be more difficult than intensity discrimination. Whereas in Experiment 1, targets and distractors were aligned on a common supramodal dimension and go/no-go discrimination mainly involved suppression of the weak distractor stimuli, Experiment 2 required identification of target stimuli in at least one modality. 


\section{Method}

Participants. Three participants took part in the second experiment (all of them female; mean age, 31 years). The participants again were students at the University of Regensburg. For their participation, they received course credit or $€ 7$ per hour. Each participant was tested in four sessions, each session lasting $3 \mathrm{~h}$. Breaks were given at a regular interval and on request of the participants.

Stimuli and Task. Timing and procedure were identical to those in Experiment 1. The visual no-go (v) stimulus was a pair of Gabor patches tilted $30^{\circ}$ to the left, of the same intensity as the visual go (V) stimulus (a pair of Gabors tilted $60^{\circ}$ to the right). The auditory go stimulus was a high-frequency tone $(\mathrm{A}, 880 \mathrm{~Hz})$, and the no-go stimulus was a low-frequency tone (a, $440 \mathrm{~Hz}$ ), both of an intensity of about $45 \mathrm{dBA}$. In the simple response task, the participants had to respond to all the stimuli. In the go/no-go task, the participants were asked to respond to target stimuli of either modality (A, V, AV, $\mathrm{Av}, \mathrm{aV})$ and to withhold their response if only nontargets were delivered (a, v, av; i.e., a low-frequency tone accompanied by a Gabor tilted $30^{\circ}$ to the left).

Race model inequality and model fit. For the simple task, RTs observed for the four different conditions ( $\mathrm{AV}, \mathrm{Av}, \mathrm{aV}$, av) were pooled for the test of the race model, as well as for parameter adjustment and goodness-of-fit test of the diffusion superposition model. This resulted in $N=400$ trials for each of the 11 mean RTs for the bimodal conditions and 200 trials for the pooled unimodal conditions (A, a, V, v). A standard diffusion superposition model with five parameters $\left(\mu_{\mathrm{A}}, \sigma_{\mathrm{A}}^{2}, \mu_{\mathrm{V}}, \sigma_{\mathrm{V}}^{2}, \mu_{\mathrm{M}}\right)$ was adjusted to the 13 SOA-specific mean RTs of the simple task, resulting in $8 d f$ s for the goodness-of-fit $\chi^{2}$ value. The corresponding model was adjusted to the mean RTs for single and redundant targets (A, V, AV; $N=100$ trials per condition) in the go/no-go task.

As in Experiment 1, a common model was fitted simultaneously describing the mean RTs for the simple response task and the go/ no-go task. For both tasks, only the mean RTs observed in conditions $\mathrm{A}, \mathrm{V}$, and $\mathrm{AV}$ were used, resulting in $2 \times 13$ conditions, with $N=100$ replications each. Using the pooled set of RTs recorded in the simple task (with 400 trials per SOA) would bias the fit of the common model toward the means observed in the simple task. Assuming, as in Experiment 1, identical elementary diffusion processes related to stimulus detection $\left(\mu_{\mathrm{A}}, \sigma_{\mathrm{A}}^{2}, \mu_{\mathrm{V}}, \sigma_{\mathrm{V}}^{2}\right)$ but separate evidence criteria and residuals for the two tasks $\left(c_{\mathrm{S}}=100, c_{\mathrm{G}}, \mu_{\mathrm{M}, \mathrm{S}}\right.$, $\mu_{\mathrm{M}, \mathrm{G}}$, for $\mathrm{S}=$ simple and $\mathrm{G}=$ go/no-go, respectively), this combined model describes 26 mean RTs using seven free parameters, resulting in $19 d f$ s for the goodness-of-fit test.

\section{Results}

For both tasks, mean RTs for the different SOAs again showed a wing-shaped pattern, with fastest responses for synchronous stimuli (see supplemental Tables S3 and S4 for the simple and go/no-go task results, respectively; see also the left panels of Figure 3). In the go/no-go task, mean RTs were substantially increased, as compared with those observed in Experiment 1. This indicates that go/ no-go discrimination was indeed more difficult than in the first experiment. Contrary to the results observed in the go/no-go task of Experiment 1, the curvature of the function SOA $\rightarrow$ mean RT was nearly zero for Participants 2 and 3 .

In both tasks, the number of false alarms was fairly low (Tables S3 and S4). Overall performance, however, decreased during sessions, as reflected by positive regression slopes of block number on overall mean RT (Participant 1, -0.7 and $7.0 \mathrm{msec}$; Participant 2, 6.0 and $7.0 \mathrm{msec}$; Participant 3, 1.4 and $1.3 \mathrm{msec}$, for simple responses and go/ no-go, respectively).
Test of the race model. In the simple response task (Inequality 2), violations of the race model were observed for Participants 1 and 3 (Table 3, Figure 4). In the go/ no-go task (Inequality 3), a significant violation of the race model inequality was observed for Participant 2 only.

Diffusion superposition model. As in Experiment 1, the diffusion superposition model was first adjusted to the mean RTs observed in the simple response task. Fitted parameters and goodness of fit for each participant are shown in Table 4 (left column). For all the participants together, the overall goodness-of-fit value was $\chi^{2}(24)=$ $41.19, p=.016$. Due to pooling $(N=400$ responses per mean in the bimodal conditions, 200 responses in the unimodal conditions), the sensitivity of the goodness-offit test for tiny deviations from the model prediction is greatly increased. Taking this into account, the goodness of fit for the model predicting the mean RTs in the simple response task with $p=.016$ still seems admissible (Figure 3, left panels).

For the go/no-go task, only RTs for the redundant condition AV were used. The results of the model fit are summarized in Table 4 (middle column). The goodness of fit of this go/no-go-specific model seems excellent $\left[\chi^{2}(24)=20.39, p=.674\right.$; see Figure 3, left panels]. An elevated criterion $c$ was chosen, resulting from the common model of simple and go/no-go responses (see below). Using this elevated $c$, the drift rates $\mu_{\mathrm{A}}$ and $\mu_{\mathrm{V}}$ observed for go/no-go roughly correspond to those observed in the simple response task. The variance parameters $\sigma_{\mathrm{A}}^{2}$ and $\sigma_{\mathrm{V}}^{2}$ and the mean residual $\mu_{\mathrm{M}}$, however, substantially differ between the two tasks. Particularly for Participants 2 and $3, \sigma_{\mathrm{A}}^{2}$ is very small, reflecting the nearly linear relationship between SOA and mean RT for audition-first stimuli (Schwarz, 1994, p. 510). For all the participants, the mean residual $\mu_{\mathrm{M}}$ is substantially higher in the go/ no-go task than in the simple response task.

Similar to Experiment 1, a common model was adjusted to the mean RTs observed in the redundant conditions of both tasks. The results are summarized in Table 4 (right column) and in Figure 3 (right panels). As compared with the simple task, both the evidence criterion $c$ and the mean residual $\mu_{\mathrm{M}}$ are increased in the go/no-go task. The fit of this common model is excellent for Participants 1 and 2 but poor for Participant 3 ( $p=.001)$. Inspection of Figure 3 (right panels) raises further doubt as to the validity of a common superposition model for Participant 3: In the go/no-go condition, mean RTs for audition-first stimuli $\mathrm{A}(\tau) \mathrm{V}$ seem to be systematically underestimated by the model, whereas mean RTs for vision-first stimuli $\mathrm{V}(\tau) \mathrm{A}$ seem to be systematically too high.

\section{Discussion}

The main question addressed in Experiment 2 was whether behavior in the go/no-go task can be described by a coactivation model if discrimination is more difficult than just bimodal intensity discrimination. In the simple response task, the parallel race model was again rejected (Table 3), and redundancy gains were adequately explained by the diffusion superposition model (Schwarz, 1994). 
Separate Fit (Participant 1)

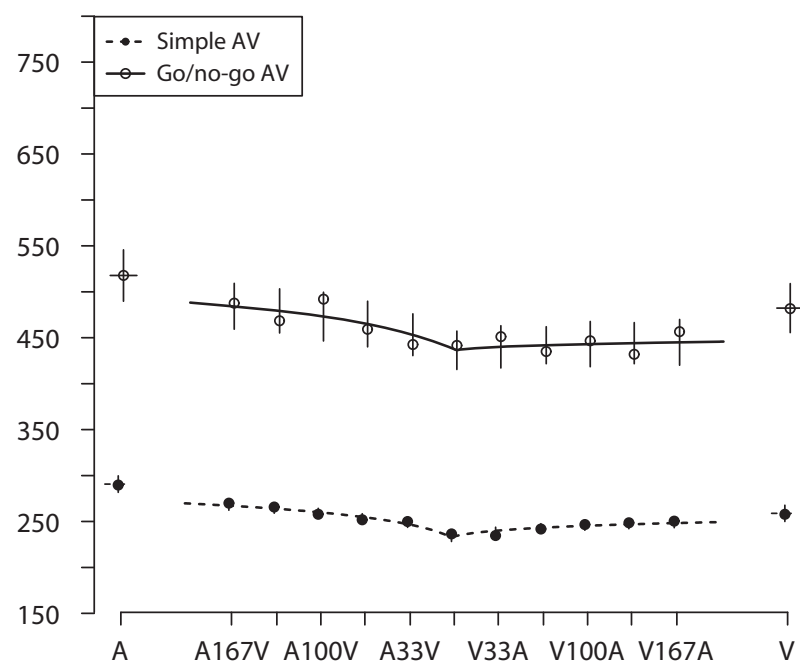

Participant 2

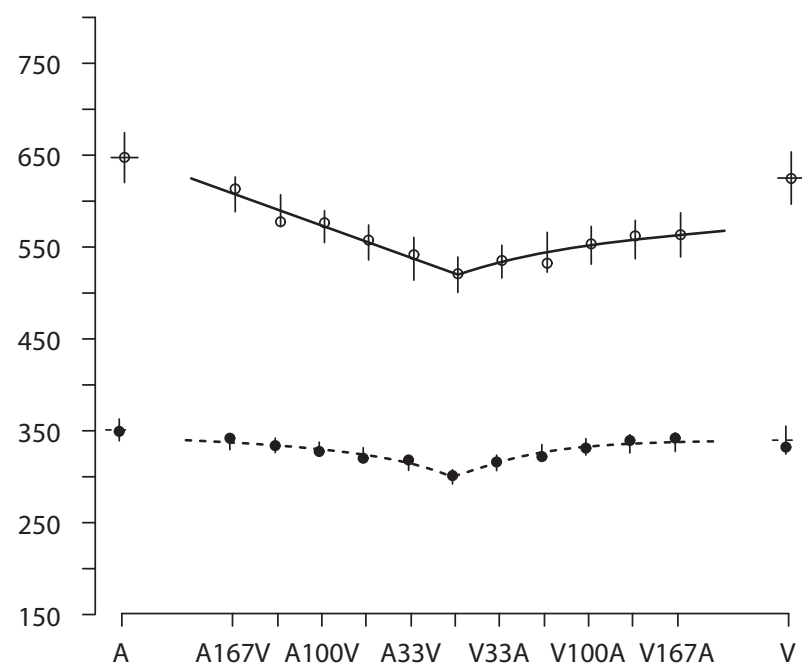

Participant 3

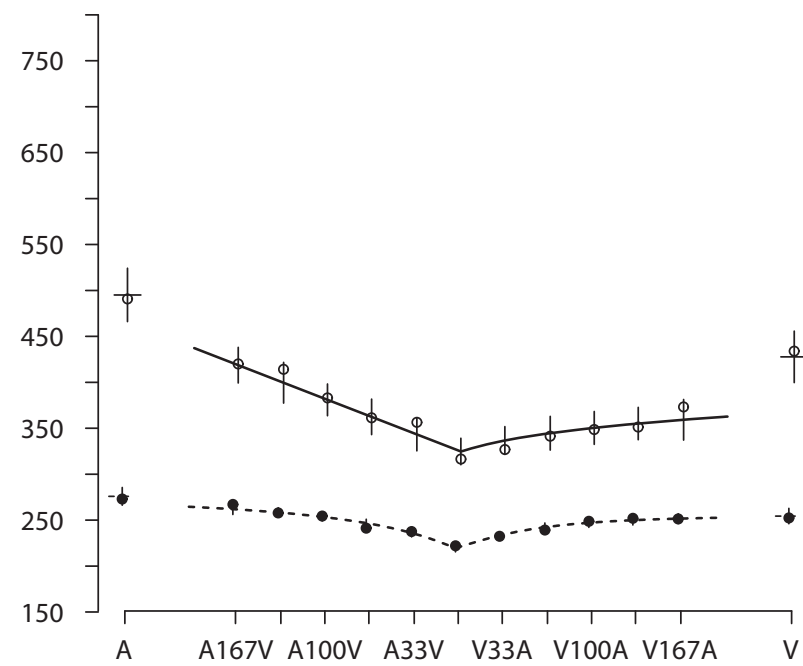

Common Model (Participant 1)

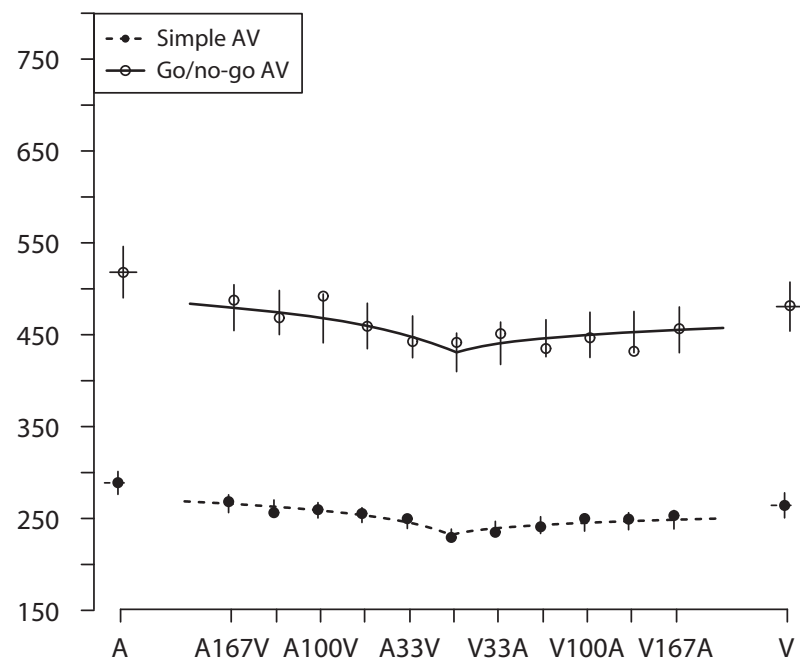

Participant 2

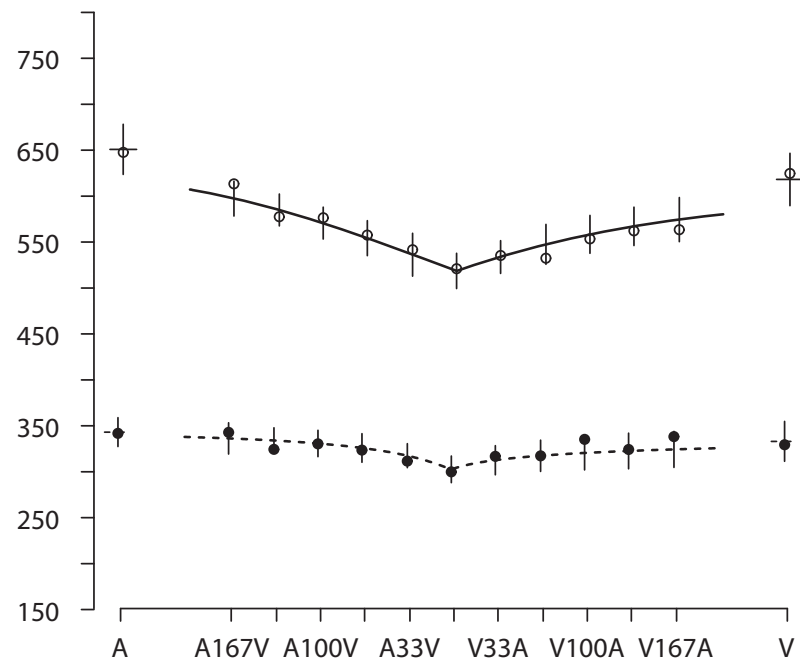

Participant 3

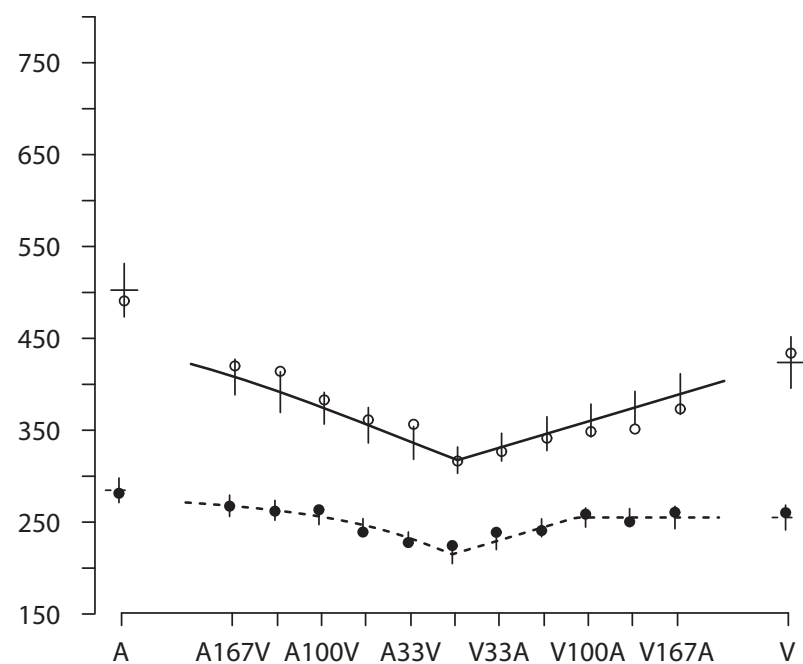

Figure 3. Left panels: Separate diffusion superposition models for simple and go/no-go responses to redundant targets in Experiment 2. Right panels: Common model for the AV conditions of the two tasks with task-specific evidence barrier and residual. 
Table 3

Experiment 2: Test of the Race Model ( $p$ Values)

\begin{tabular}{crccc}
\hline & \multicolumn{3}{c}{ Participant } & \\
\cline { 2 - 4 } Task & 1 & 2 & 3 & Summary \\
\hline Simple & $<.001$ & .454 & .005 & $<.001$ \\
Go/no-go & .404 & .010 & .113 & .018 \\
\hline
\end{tabular}

The increased complexity of the go/no-go task in Experiment 2 is reflected by substantially higher mean RTs, as compared with those observed in the intensity discrimination task in Experiment 1 (e.g., mean go/no-go RTs for AV in Experiment 1 were 301, 273, $307 \mathrm{msec}$; mean go/no-go RTs for AV in Experiment 2 were 442, 521, 316 msec). Model fit for mean RTs observed for redundant targets in the go/no-go task was excellent, although some diffusion variance parameters had unusually low values and the function relating SOA and mean RT was nearly linear. In fact, both race models (Ulrich \& Miller, 1997) and "standard" diffusion superposition models (Schwarz, 1989, 1994) predict a wing-shaped relationship between SOA and mean RT (e.g., Schwarz, 1994, Figure 1).

As in Experiment 1, a common model was adjusted to the two tasks, with common parameters describing modality-specific buildup of evidence but task-specific evidence barriers and residuals. The fit of this common model was excellent for Participants 1 and 2. The diffusion processes describe the subset of task-unspecific perceptual processes common to both tasks, whereas the evidence criterion and the residual were increased in the go/no-go condition (Table 4, right columns; e.g., Participant 1: simple, $c_{\mathrm{S}}=100, \mu_{\mathrm{M}, \mathrm{S}}=194 \mathrm{msec} ;$ go/no-go, $c_{\mathrm{G}}=153, \mu_{\mathrm{M}, \mathrm{G}}=$ $375 \mathrm{msec}$ ). On about $50 \%$ of the trials for the go/no-go task, targets and distractors were presented together (conditions $\mathrm{Av}, \mathrm{aV}$ ). These conditions cause response conflicts. The higher $\mu_{\mathrm{M}}$ observed in the go/no-go task suggests that Participants 1 and 2 solved these response conflicts, at least partially, at the level of $\mathbf{M}$. The overall fit of this common model was not satisfactory, however, mainly due to the poor fit of Participant 3. Either this participant adopted different strategies for the two tasks, or modality-specific buildup of evidence occurred at different speeds in the two tasks.

Response competition effects are a common issue in redundant-signals experiments with go/no-go stimuli (e.g., Fournier \& Eriksen, 1990; Grice, Canham, \& Gwynne, 1984, p. 565). Consider the present experimental setup with targets (A, V) and nontargets (a, v) in two modalities, which has become a standard setup in EEG studies on multisensory integration (audiovisual oddball task; e.g., Gondan et al., 2005; Schröger \& Widmann, 1998; Teder-Sälejärvi, Di Russo, McDonald, \& Hillyard, 2005; Teder-Sälejärvi et al., 2002). In such experiments, the participant has to respond if any target appears - that is, in conditions $\mathrm{AV}, \mathrm{Av}, \mathrm{aV}$ - whereas in condition av, the participant has to withhold the response. Combinations of targets and nontargets, however, might cause response competition similar to the incompatibility effects observed in flanker tasks (e.g., Eriksen \& Hoffman, 1973). Thus, it could be argued that on about $50 \%$ of the trials, the par-
Race Model Test (Participant 1)

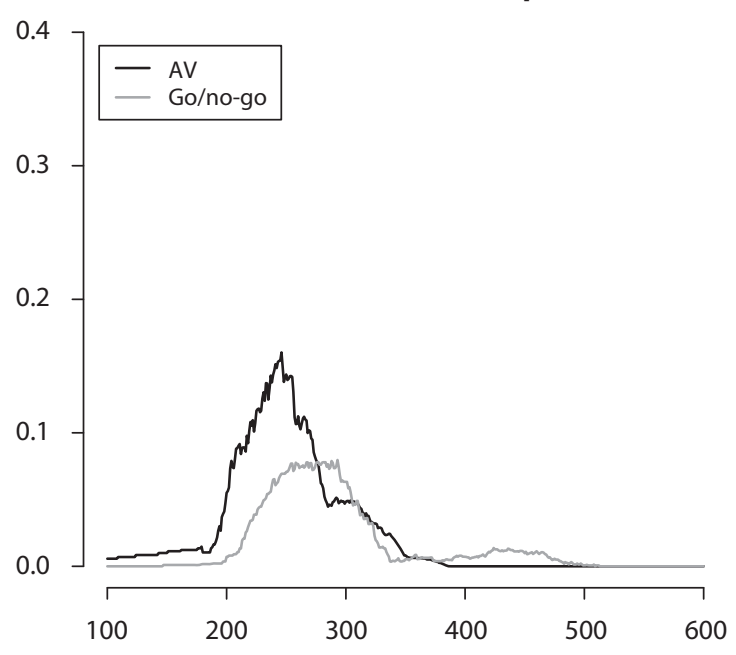

Participant 2

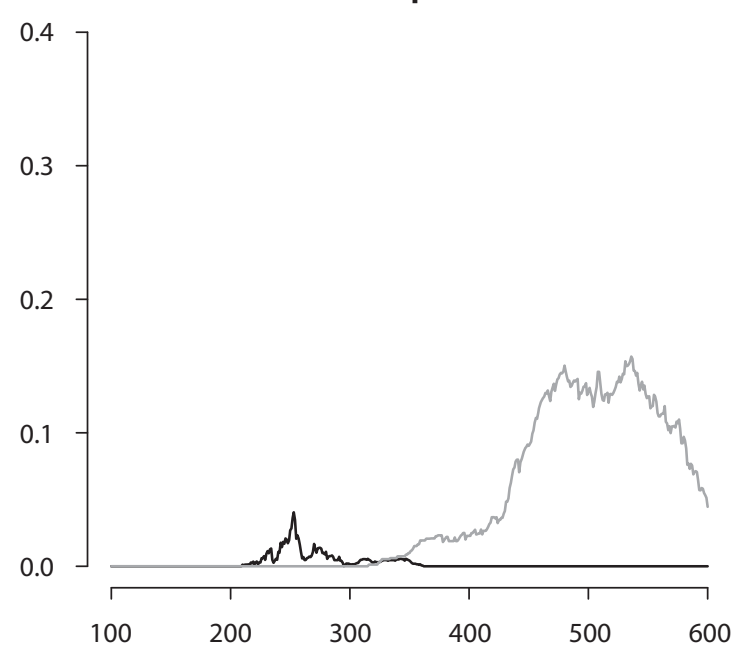

Participant 3

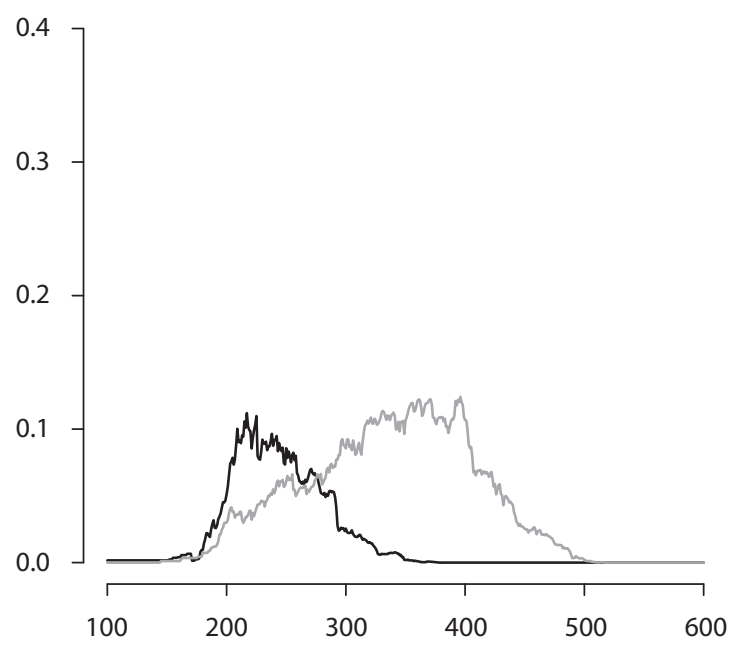

Figure 4. Experiment 2: Test of the race model inequality. For significance tests, see Table 3. 
Table 4

Experiment 2: Diffusion Superposition Model for Simple Responses and Go/No-Go Responses to AV and Common Model for Both Tasks

\begin{tabular}{|c|c|c|c|c|c|c|c|c|c|}
\hline & \multicolumn{3}{|c|}{ Simple } & \multicolumn{3}{|c|}{ Go/No-Go AV } & \multicolumn{3}{|c|}{ Simple AV + Go/No-Go AV } \\
\hline & $\begin{array}{c}\text { Participant } \\
1\end{array}$ & $\begin{array}{c}\text { Participant } \\
2\end{array}$ & $\begin{array}{c}\text { Participant } \\
3\end{array}$ & $\begin{array}{c}\text { Participant } \\
1\end{array}$ & $\begin{array}{l}\text { Participant } \\
2\end{array}$ & $\begin{array}{c}\text { Participant } \\
3\end{array}$ & $\begin{array}{c}\text { Participant } \\
1\end{array}$ & $\begin{array}{c}\text { Participant } \\
2\end{array}$ & $\begin{array}{c}\text { Participant } \\
3\end{array}$ \\
\hline$\mu_{\mathrm{V}}$ & 1.55 & 1.18 & 1.26 & 1.43 & 1.48 & 1.13 & 1.33 & 1.52 & 1.08 \\
\hline$\sigma_{\mathrm{V}}^{2}$ & $1,207.1$ & 50.3 & 85.5 & 19,104 & $1,913.5$ & $2,101.9$ & $1,301.8$ & 590.8 & 0.10 \\
\hline$\mu_{\mathrm{A}}$ & 1.04 & 1.04 & 1.00 & 1.08 & 1.34 & 0.88 & 1.00 & 1.32 & 0.82 \\
\hline$\sigma_{\mathrm{A}}^{2}$ & 460.4 & 200.2 & 156.1 & 404.4 & 0.63 & 5.3 & 404.8 & 198.0 & 84.0 \\
\hline$c_{\mathrm{S}}^{\mathrm{A}}$ & $(100)$ & $(100)$ & $(100)$ & - & - & - & $(100)$ & $(100)$ & $(100)$ \\
\hline$\mu_{\mathrm{M}, \mathrm{S}}$ & 194.2 & 254.8 & 175.3 & - & - & - & 188.9 & 267.5 & 162.2 \\
\hline$c_{\mathrm{G}}$ & - & - & - & $(153.4)$ & $(326.7)$ & $(265.6)$ & 153.4 & 326.7 & 265.6 \\
\hline$\mu_{\mathrm{M}, \mathrm{G}}$ & - & - & - & 375.3 & 404.1 & 192.2 & 364.9 & 403.8 & 177.1 \\
\hline GOF $\chi^{2}$ & 19.20 & 7.00 & 14.99 & 7.47 & 4.05 & 8.88 & 19.37 & 15.97 & 45.46 \\
\hline$d f$ & 8 & 8 & 8 & 8 & 8 & 8 & 19 & 19 & 19 \\
\hline$p$ & .014 & .537 & .059 & .487 & .853 & .353 & .433 & .659 & .001 \\
\hline Summary & \multicolumn{3}{|c|}{$\chi^{2}(24)=41.19, p=.016$} & \multicolumn{3}{|c|}{$\chi^{2}(24)=20.39, p=.674$} & \multicolumn{3}{|c|}{$\chi^{2}(57)=80.80, p=.021$} \\
\hline
\end{tabular}

Note-Fixed parameters are given in parentheses. GOF, goodness of fit.

ticipants had to cope with response conflicts induced by the target/nontarget combination. Since the presentation of the stimuli was in randomized order, the participants could not foresee whether the upcoming stimulus pair was conflicting (Av, aV) or not (AV, av). Participant 3 might, therefore, have adopted a conservative strategy to handle response competition. For example, the auditory component is processed first. Depending on whether it is a target, processing stops, and a response is initiated. The visual component is processed only if the auditory stimulus was a nontarget on a given trial.

\section{SERIAL SELF-TERMINATING MODEL}

In contrast to Experiment 1, the modality-specific target stimuli (right-tilted Gabor, high tone) of Experiment 2 did not share common supramodal features such as intensity. May coactivation occur under such circumstances? In difficult tasks such as the go/no-go discrimination task of Experiment 2, serial processing seems to be a plausible alternative to parallel and coactivation models (Luria \& Meiran, 2005; Townsend \& Nozawa, 1997). In the serial self-terminating model, one stimulus component is processed after the other; processing stops as soon as a target has been identified. For illustration, consider a simple response task with redundant AV stimuli presented at different onset asynchronies (the same argument holds for the redundant condition of the go/ no-go task). Because both components are targets, processing always stops after the first stimulus component. Therefore, the serial self-terminating model reduces to a mixture model of auditory and visual processing times (e.g., Schweickert, Giorgini, \& Dzhafarov, 2000, p. 506). Assume that, on each trial, with probability $\pi$, the visual stimulus is processed first. With probability $1-\pi$, the auditory stimulus is processed first. Let $\mathbf{D}_{\mathrm{A}}$ denote the processing time for the auditory component and $\mathbf{D}_{\mathrm{V}}$ for the visual component. Assuming again that RT $\mathbf{T}$ is the sum of the processing time $\mathbf{D}$ and a residual $\mathbf{M}$ (e.g., motor processes) with constant expectation $\mu_{\mathrm{M}}$, the mean
RTs for bimodal stimuli presented with onset asynchrony are easily derived:

$$
\begin{aligned}
\mathrm{E}\left[\mathbf{T}_{\mathrm{AV}}\right] & =\mu_{\mathrm{M}}+\pi \mathrm{E}\left[\mathbf{D}_{\mathrm{V}}\right]+(1-\pi) \mathrm{E}\left[\mathbf{D}_{\mathrm{A}}\right] \\
\mathrm{E}\left[\mathbf{T}_{\mathrm{A}(\tau) \mathrm{V}}\right] & =\mu_{\mathrm{M}}+\pi\left(\tau+\mathrm{E}\left[\mathbf{D}_{\mathrm{V}}\right]\right)+(1-\pi) \mathrm{E}\left[\mathbf{D}_{\mathrm{A}}\right] \\
& =\mathrm{E}\left[\mathbf{T}_{\mathrm{AV}}\right]+\pi \times \tau .
\end{aligned}
$$

Similarly, $\mathrm{E}\left[\mathbf{T}_{\mathrm{V}(\tau) \mathrm{A}}\right]=\mathrm{E}\left[\mathbf{T}_{\mathrm{AV}}\right]+(1-\pi) \times \tau$. Thus, the mixture model predicts a linear relationship between SOA and mean RT for both audition-first and vision-first stimuli, with the slopes of the two functions $\tau \rightarrow \mathrm{E}\left[\mathbf{T}_{\mathrm{A}(\tau) \mathrm{V}}\right]$ and $\tau \rightarrow \mathrm{E}\left[\mathbf{T}_{\mathrm{V}(\tau) \mathrm{A}}\right]$ adding up to one. ${ }^{1}$

In the serial self-terminating model, the probability $\pi$ for processing the visual component first can be assumed to be constant for a limited range of SOAs. For small SOAs, it can even be beneficial to schedule, in advance, which modality to attend in the upcoming trial (e.g., De Jong, 1995). For large SOAs, however, this strategy might fail: If, for example, the participant attends the auditory channel in V167A, processing starts with a substantial delay of $167 \mathrm{msec}$. If the system is more flexible - that is, $\pi$ varies over the SOA range - a model assuming constant $\pi$ overestimates the mean RTs observed at large onset asynchronies. Thus, additional assumptions are needed for these conditions (e.g., Equation 8; see below). Unimodal stimuli can be considered an even more extreme case with infinite onset asynchrony: $\mathrm{A}=\mathrm{A}(\tau) \mathrm{V}$ with $\tau=\infty, \mathrm{V}=\mathrm{V}(\tau) \mathrm{A}$ with $\tau=\infty$. For unimodal stimuli, the serial model is mute; in fact, for $\tau=\infty$, Equation 7 predicts infinite mean RTs.

Interestingly, the relationship described by Equation 7 can be mimicked by a diffusion superposition model with zero diffusion variance parameters $\sigma_{\mathrm{A}}^{2}=\sigma_{\mathrm{V}}^{2}=0$. With zero variance, buildup of activation in AV occurs, each millisecond, by $\mu_{\mathrm{A}}+\mu_{\mathrm{V}}$ until the barrier $c$ has been reached, such that $\mathbf{D}_{\mathrm{AV}}=c /\left(\mu_{\mathrm{A}}+\mu_{\mathrm{V}}\right)$. What happens in $\mathrm{A}(\tau) \mathrm{V}$ ? We assume for a moment that the onset asynchrony $\tau$ is small, such that the evidence criterion has not been reached before the onset of the second stimulus component. As compared with the synchronous condition $\mathrm{AV}$, passage of the barrier $c$ is delayed because, for the 
Table 5

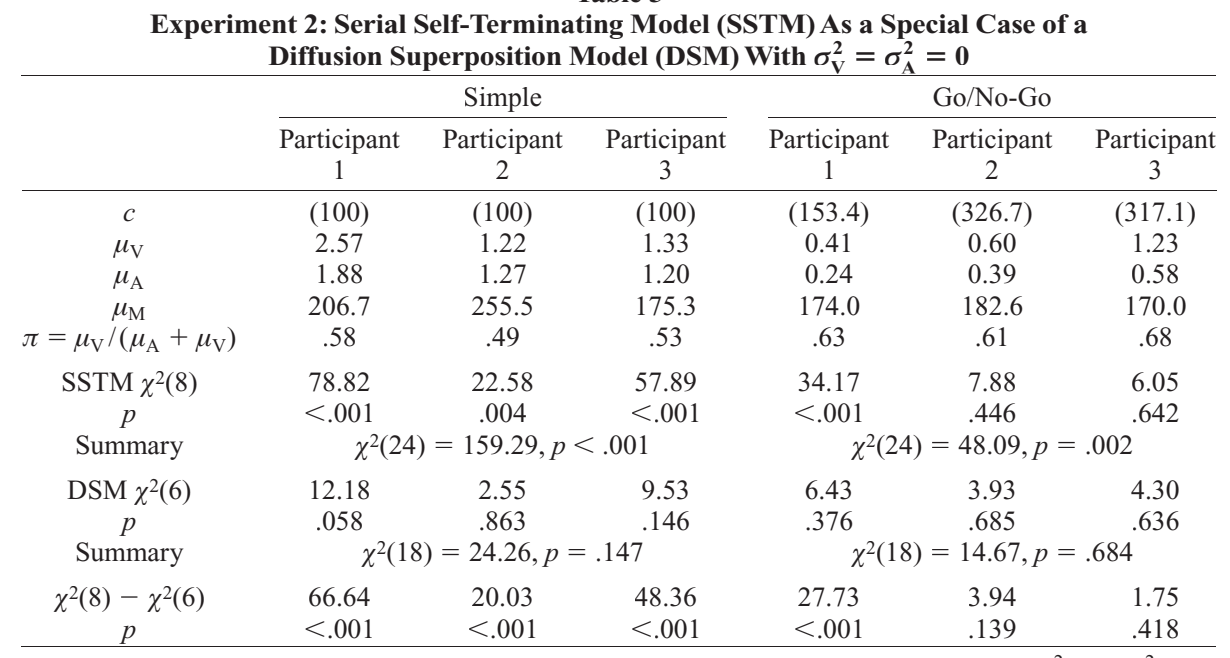

Note-Fixed parameters are given in parentheses. Results for the unrestricted model (DSM, $\sigma_{\mathrm{V}}^{2}>0, \sigma_{\mathrm{A}}^{2}>0$ ) are shown for comparison.

first $\tau$ milliseconds, buildup of evidence occurs only with drift $\mu_{\mathrm{A}}$, instead of $\mu_{\mathrm{A}}+\mu_{\mathrm{V}}$. Equivalently, when processing of AV has finished at time $c /\left(\mu_{\mathrm{A}}+\mu_{\mathrm{V}}\right)$, a remaining activation of $\mu_{\mathrm{V}} \times \tau$ needs to be attained for $\mathrm{A}(\tau) \mathrm{V}$, at rate $\mu_{\mathrm{A}}+\mu_{\mathrm{V}}$ :

$$
\begin{aligned}
& \mathrm{E}\left[\mathbf{T}_{\mathrm{AV}}\right]=\mu_{\mathrm{M}}+\mathrm{E}\left[\mathbf{D}_{\mathrm{AV}}\right]=\mu_{\mathrm{M}}+c /\left(\mu_{\mathrm{A}}+\mu_{\mathrm{V}}\right) \\
& \mathrm{E}\left[\mathbf{T}_{\mathrm{A}(\tau) \mathrm{V}} \mid \tau \text { small }\right]=\mathrm{E}\left[\mathbf{T}_{\mathrm{AV}}\right]+\mu_{\mathrm{V}} /\left(\mu_{\mathrm{A}}+\mu_{\mathrm{V}}\right) \times \tau .
\end{aligned}
$$

Similarly, $\mathrm{E}\left[\mathbf{T}_{\mathrm{V}(\tau) \mathrm{A}} \mid \tau\right.$ small $]=\mathrm{E}\left[\mathbf{T}_{\mathrm{AV}}\right]+\mu_{\mathrm{A}} /\left(\mu_{\mathrm{A}}+\right.$ $\left.\mu_{\mathrm{V}}\right) \times \tau$. If $\mu_{\mathrm{V}} /\left(\mu_{\mathrm{A}}+\mu_{\mathrm{V}}\right)$ is substituted by $\pi$ and $\mu_{\mathrm{A}} /\left(\mu_{\mathrm{A}}+\mu_{\mathrm{V}}\right)$ is substituted by $1-\pi$, the serial selfterminating model described by Equation 7 numerically corresponds to a diffusion superposition model with zero variance parameters.

For larger SOAs, the barrier $c$ has already been reached before the onset of the second stimulus. In other words, $\mathrm{E}\left[\mathbf{T}_{\mathrm{A}(\tau) \mathrm{V}} \mid \tau\right.$ large $]$ cannot exceed $\mathrm{E}\left[\mathbf{T}_{\mathrm{A}}\right]$ :

$$
\mathrm{E}\left[\mathbf{T}_{\mathrm{A}(\tau) \mathrm{V}}\right]=\min \left\{\mathrm{E}\left[\mathbf{T}_{\mathrm{A}}\right], \mathrm{E}\left[\mathbf{T}_{\mathrm{A}(\tau) \mathrm{V}} \mid \tau \text { small }\right]\right\} .
$$

We have noted above that the serial self-terminating model (Equation 7) breaks down for large SOAs. The upper bound given by Equation 8 seems, thus, to be a reasonable ad hoc choice for the serial self-terminating model as well. To summarize, from a technical perspective, the serial selfterminating model with constant $\pi$ might be considered a special case of a diffusion superposition model with $\sigma_{\mathrm{A}}^{2}=$ $\sigma_{\mathrm{V}}^{2}=0$ (i.e., a deterministic model). This model inclusion allows the direct comparison of the goodness of fit of the two models using standard $\chi^{2}$ technique.

Interestingly, and in contrast to serial exhaustive models (Townsend \& Nozawa, 1997), the serial self-terminating model does not predict a violation of the race model inequality (see Appendix B).

\section{Test of the Serial Model}

The serial self-terminating model (Equations 7 and 8) was first tested for the simple response task and for the redundant conditions (AV) of the go/no-go task. We have noted above that the serial model is mute with respect to unimodal stimuli; for this reason, unimodal conditions were not included in the model fit. In a first step, the standard diffusion superposition model was adjusted to the mean RTs observed for the 11 finite SOA conditions; in the second step, the serial self-terminating model was fitted by means of a diffusion superposition model with zero diffusion variance parameters, $\sigma_{\mathrm{A}}^{2}=\sigma_{\mathrm{V}}^{2}=0$. The results are summarized in Table 5 (Figure 5, left panels).

For the simple response task, it is immediately evident that the serial model fails in all participants, which is also reflected by a huge overall goodness-of-fit value $\left[\chi^{2}(24)=159.29, p<.001\right]$. Releasing the diffusion variance parameters (i.e., adjusting the standard diffusion superposition model) considerably improves the overall goodness of fit $\left[\chi^{2}(18)=24.26, p=.147\right]$. As was mentioned above, the two models are nested, such that the models can be compared with each other: In all participants, the difference between the two goodness-offit values is significant $\left[\chi^{2}(8)-\chi^{2}(6)=66.64,20.03\right.$, and 48.36, respectively; all $p \mathrm{~s}<.001]$. Technically, this result indicates that the variance parameters are significantly greater than zero, which statistically rejects the serial self-terminating model.

Inconsistent results are obtained for the go/no-go task. For Participant 1, the serial model fails again. For Participants 2 and 3, however, the mean RTs support a serial selfterminating model (Participant 2, $\chi^{2}=7.88, p=.446$; Participant $3, \chi^{2}=6.05, p=.642$ ). For these 2 participants, comparison with the unrestricted diffusion superposition model shows that releasing the variance parameters does not significantly improve the fit [Participant 2, $\chi^{2}(8)-\chi^{2}(6)=3.94, p=.139$; Participant $3, \chi^{2}(8)-$ $\left.\chi^{2}(6)=1.75, p=.418\right]$.

As is shown in Appendix B, the serial self-terminating model predicts that the race model inequality holds. The RT distributions of Participant 2 show a violation of the 
Separate SSTMs (Participant 1)

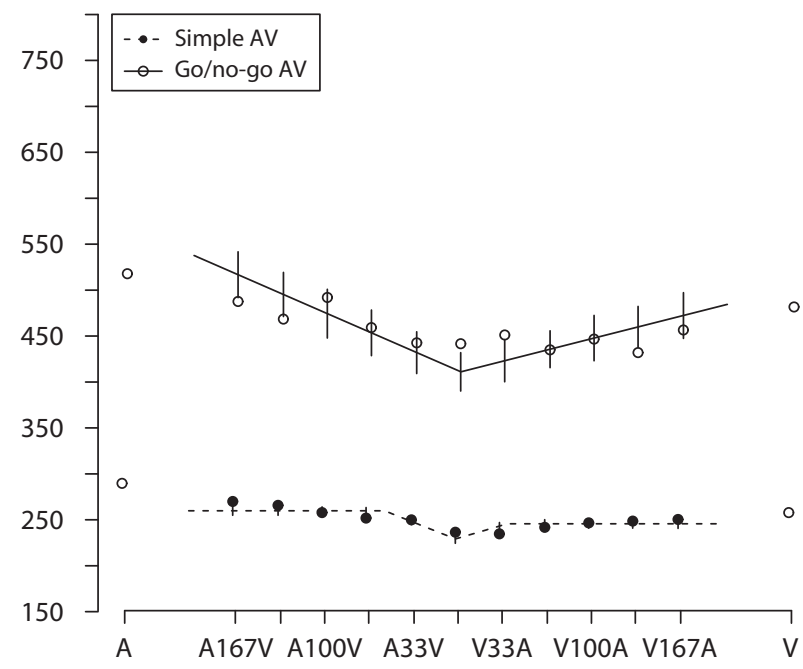

Participant 2

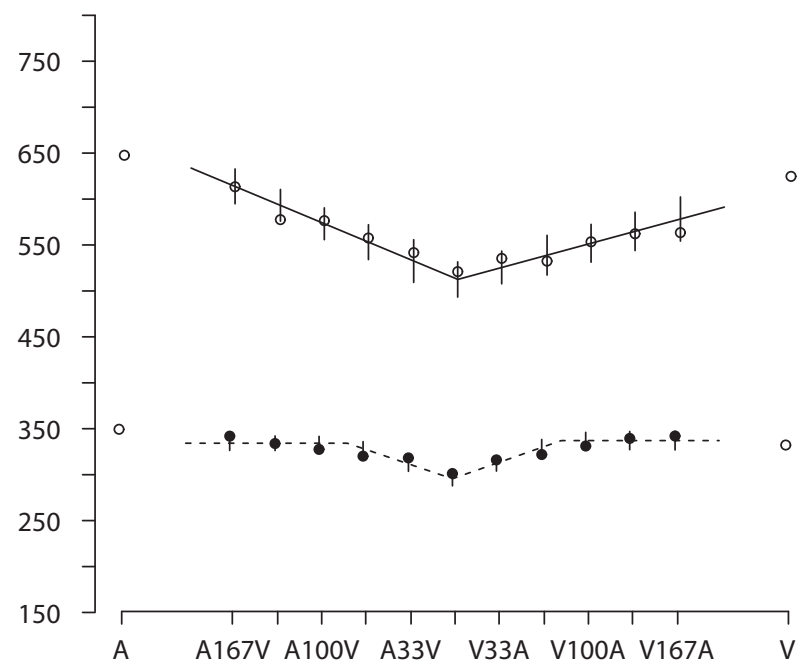

Participant 3

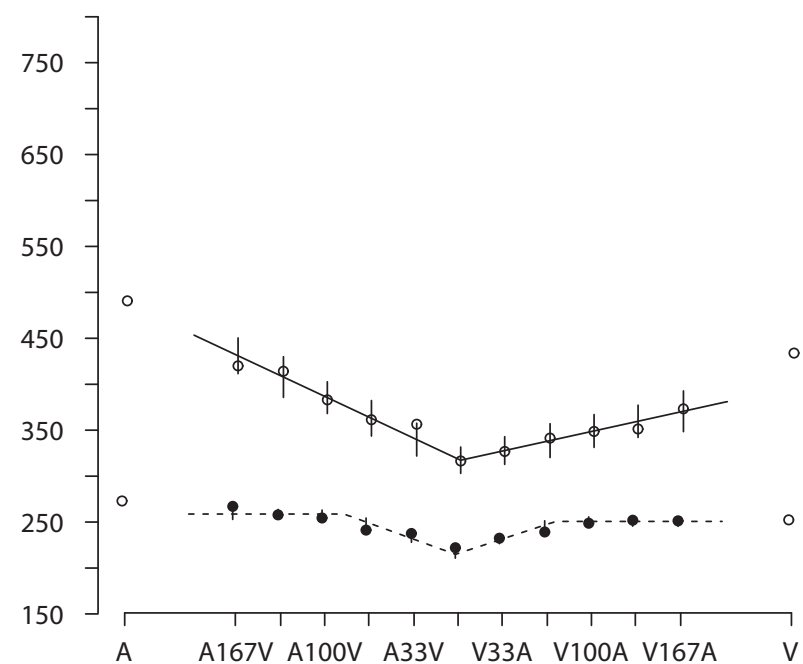

Go/No-Go (Participant 1)

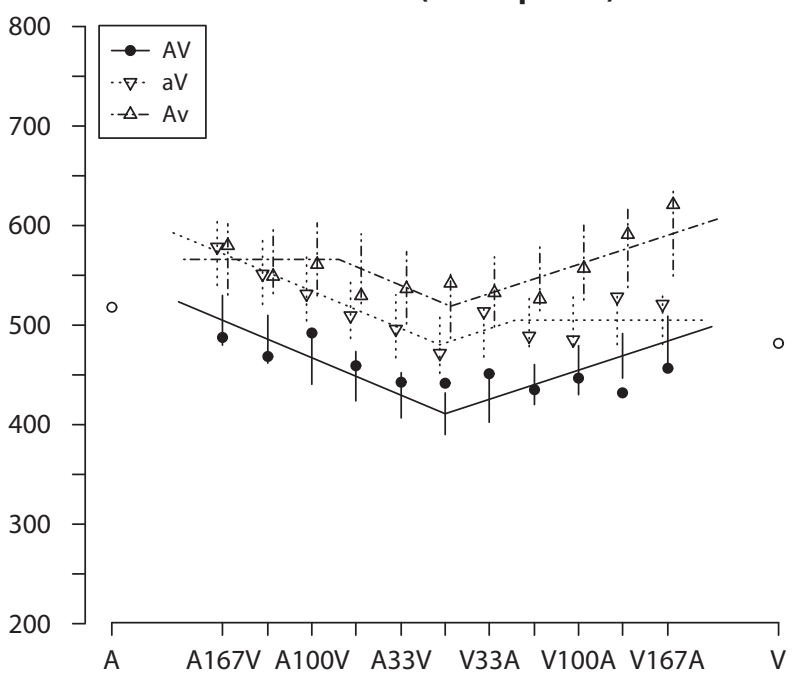

Participant 2

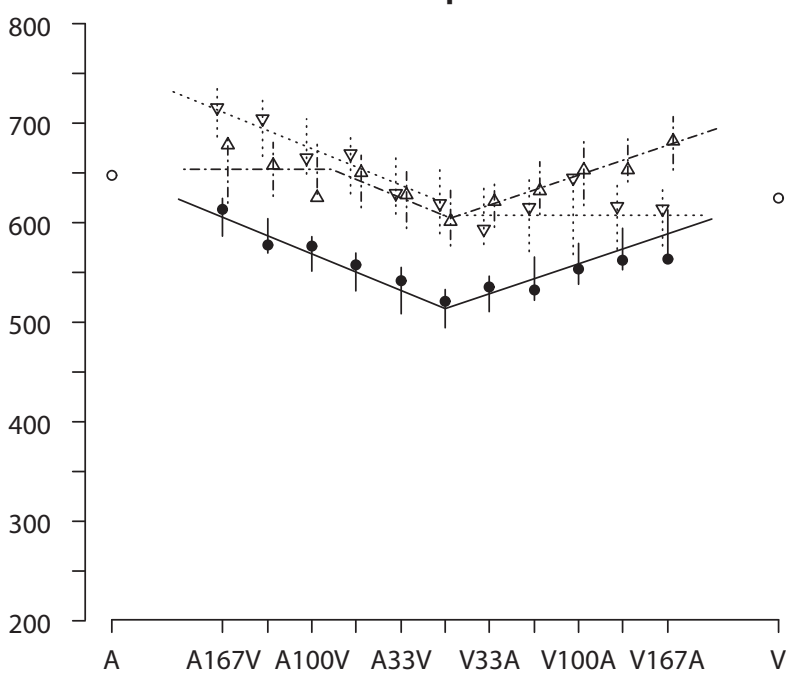

Participant 3

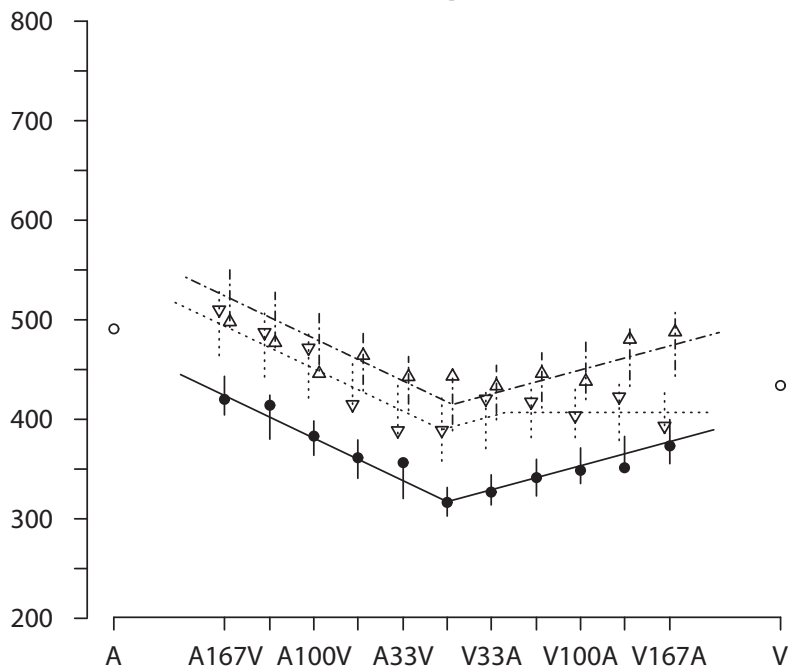

Figure 5. Left panels: Serial self-terminating model (SSTM) for simple and go/no-go responses to AV in Experiment 2. Right panels: Serial model for go/no-go task including redundant targets and combinations of target and distractors. 
race model inequality, which rejects the serial model. In contrast, the RT distributions observed for Participant 3 are consistent with Inequality 3 ; we can, thus, conclude that Participant 3 coped with the more complex requirements of the go/no-go task by serially processing the two sources of information.

\section{Responses to Nonredundant Targets}

For combinations of targets and nontargets, we first consider the two conditions $\mathrm{Av}$ and $\mathrm{A}(\tau) \mathrm{V}$, with lowercase letters denoting the nontargets. With probability $1-\pi$, the auditory target component is processed first, directly eliciting the response. With probability $\pi$, the visual distractor is processed first. Since the visual component $\mathrm{v}$ is a nontarget, the auditory component is processed next, and a response is initiated:

$$
\begin{aligned}
\mathrm{E}\left[\mathbf{T}_{\mathrm{Av}}\right]= & \mu_{\mathrm{M}}+(1-\pi) \mathrm{E}\left[\mathbf{D}_{\mathrm{A}}\right] \\
& +\pi\left(\mathrm{E}\left[\mathbf{D}_{\mathrm{v}}\right]+\mathrm{E}\left[\mathbf{D}_{\mathrm{A}}\right]\right) \\
\mathrm{E}\left[\mathbf{T}_{\mathrm{A}(\tau) \mathrm{v}}\right]= & \mu_{\mathrm{M}}+(1-\pi) \mathrm{E}\left[\mathbf{D}_{\mathrm{A}}\right] \\
& +\pi\left(\tau+\mathrm{E}\left[\mathbf{D}_{\mathrm{v}}\right]+\mathrm{E}\left[\mathbf{D}_{\mathrm{A}}\right]\right) \\
= & \mathrm{E}\left[\mathbf{T}_{\mathrm{Av}}\right]+\pi \times \tau .
\end{aligned}
$$

Similarly, for visual targets followed by auditory distractors, the model predicts $\mathrm{E}\left[\mathbf{T}_{\mathrm{V}(\tau) \mathrm{a}}\right]=\mathrm{E}\left[\mathbf{T}_{\mathrm{aV}}\right]+(1-\pi) \times \tau$. Assuming $\pi$ to be constant over the SOA range might again be problematic for large onset asynchronies. For example, in a unimodal auditory stimulus or in $\mathrm{A} 167 \mathrm{v}$, it is clear that the participant will not eternally wait for the visual stimulus to appear. If $\pi$ is held constant in the model fit, for large onset asynchronies, Equation 9 might overestimate the mean RTs, biasing the goodness-of-fit test toward rejection of the serial model.

What happens in condition $\mathrm{a}(\tau) \mathrm{V}$, when the nontarget component is presented first? Assume again that, with probability $\pi$, the visual component is processed first. This is the target component, so that a response can directly be initiated (with delay $\tau$, though). With probability $1-\pi$, the auditory nontarget is processed first. If the time required for processing the auditory nontarget is shorter than $\tau$, the system must "wait" for the appearance of the visual target stimulus:

$$
\begin{aligned}
\mathrm{E}\left[\mathbf{T}_{\mathrm{aV}}\right]= & \mu_{\mathrm{M}}+\pi \mathrm{E}\left[\mathbf{D}_{\mathrm{V}}\right] \\
& +(1-\pi)\left(\mathrm{E}\left[\mathbf{D}_{\mathrm{a}}\right]+\mathrm{E}\left[\mathbf{D}_{\mathrm{V}}\right]\right) \\
\mathrm{E}\left[\mathbf{T}_{\mathrm{a}(\tau) \mathrm{V}}\right]= & \mu_{\mathrm{M}}+\pi\left(\tau+\mathrm{E}\left[\mathbf{D}_{\mathrm{V}}\right]\right) \\
& +(1-\pi)\left(\mathrm{E}\left[\max \left(\mathbf{D}_{\mathrm{a}}, \tau\right)\right]+\mathrm{E}\left[\mathbf{D}_{\mathrm{V}}\right]\right) \\
\mathrm{E}\left[\mathbf{T}_{\mathrm{a}(\tau) \mathrm{V}}\right] \geq & \mathrm{E}\left[\mathbf{T}_{\mathrm{aV}}\right]+\pi \times \tau .
\end{aligned}
$$

For SOAs sufficiently small, $\mathbf{D}_{\mathrm{a}}>\tau$, and equality holds. For large SOAs, however, $\mathrm{E}\left[\max \left(\mathbf{D}_{\mathrm{a}}, \tau\right)\right]>\mathrm{E}\left[\mathbf{D}_{\mathrm{a}}\right]$, and Equation 10 might underestimate the mean RT in condition $\mathrm{a}(\tau) \mathrm{V}$. For visual distractors followed by auditory target stimuli, the serial self-terminating model predicts $\mathrm{E}\left[\mathbf{T}_{\mathrm{v}(\tau) \mathrm{A}}\right] \geq \mathrm{E}\left[\mathbf{T}_{\mathrm{Av}}\right]+(1-\pi) \times \tau$.

In a last step, the serial self-terminating model was adjusted to the mean RTs of the go/no-go task, including responses to nonredundant targets. For this purpose, we
Table 6

Serial Self-Terminating Model for Go/No-Go Responses

\begin{tabular}{cccc}
\hline & \multicolumn{3}{c}{ Participant } \\
\cline { 2 - 4 }$c$ & 1 & 2 & 3 \\
$\mu_{\mathrm{V}}$ & $(157.2)$ & $(269.3)$ & $(317.1)$ \\
$\mu_{\mathrm{A}}$ & 0.73 & 1.29 & 1.28 \\
$\mu_{\mathrm{M}, \mathrm{AV}}$ & 0.57 & 1.06 & 0.72 \\
$\mu_{\mathrm{M}, \mathrm{AV}}$ & 290.2 & 399.0 & 158.7 \\
$\mu_{\mathrm{M}, \mathrm{aV}}$ & 398.2 & 489.9 & 256.4 \\
$\pi=\mu_{\mathrm{V}} /\left(\mu_{\mathrm{A}}+\mu_{\mathrm{V}}\right)$ & 359.5 & 507.0 & 231.1 \\
$\chi^{2}(28)$ & .56 & .55 & .64 \\
$p$ & 59.71 & 27.32 & 38.21 \\
Summary & $<.001$ & .501 & .095 \\
& $\chi^{2}(84)=125.24, p=.002$ \\
\hline
\end{tabular}

fitted again a deterministic diffusion superposition model (Equation 8) to the mean RTs observed for stimulus pairings $\mathrm{AV}, \mathrm{Av}$, and $\mathrm{aV}$ with finite SOAs. The system was assumed to be rigid, with constant probability $\pi=\mu_{\mathrm{V}} /$ $\left(\mu_{\mathrm{A}}+\mu_{\mathrm{V}}\right)$ for all SOAs and relevance pairings. ${ }^{2}$ Different $\mu_{\mathrm{M}} \mathrm{s}$ were allowed for conditions $\mathrm{AV}, \mathrm{Av}$, and $\mathrm{aV}$, reflecting the different mean RTs for the synchronous conditions $\left(\mathrm{E}\left[\mathbf{T}_{\mathrm{AV}}\right], \mathrm{E}\left[\mathbf{T}_{\mathrm{Av}}\right], \mathrm{E}\left[\mathbf{T}_{\mathrm{aV}}\right]\right.$; see Equations 7, 9, and 10). Five parameters are, thus, needed $\left(\mu_{\mathrm{A}}, \mu_{\mathrm{V}}, \mu_{\mathrm{M}, \mathrm{AV}}, \mu_{\mathrm{M}, \mathrm{Av}}, \mu_{\mathrm{M}, \mathrm{aV}}\right)$ to describe 33 mean RTs (11 finite SOAs per condition), resulting in $28 d f \mathrm{~s}$ for the goodness-of-fit $\chi^{2}$ value.

Table 6 shows the adjusted parameters and the results of the goodness-of-fit test. In Participant 1, the fit of the serial self-terminating model is poor $(p<.001)$, as was already seen in the responses to redundant targets (Table 5, Figure 5). In contrast, for Participants 2 and 3, the serial self-terminating model well describes the mean RTs ( $p=$ .501 and .095 , respectively), both for redundant targets and for combinations of targets and distractors. At least for Participant 3, whose RT distributions did not violate the race model inequality, the results further support the serial self-terminating model.

In the model adjustment, the probability $\pi$ for processing the visual channel first was assumed to be constant over the SOA range. This seems implausible at first glance. Why should $\pi$ be equal in A167V and V167A? Would it not be more efficient to process the two components on a first-come, first-served basis? De Jong (1995) provided a tentative answer to this issue. If the temporal order of processing is flexible, a decision on which stimulus is to be processed first must be made on each single trial. This decision might be time-consuming. Scheduling processing order before trial onset might save time. Within a limited range of SOAs, processing in a prespecified temporal order might, thus, be more efficient than processing in a flexible order. Moreover, if the participant decides in advance which modality is processed first, this modality is attended, which by itself enables faster processing of the respective stimulus component. As a side note, Participant 3 showed considerably faster responses than did the other 2 participants. It could, thus, be argued that serial processing with a prespecified schedule can be a very efficient strategy for handling the complex requirements of the go/no-go task in the present study. 


\section{GENERAL DISCUSSION}

In divided-attention tasks in which participants have to respond to stimuli from two modalities, RTs have often been observed to be shorter if both stimuli are presented at the same time. This redundant-signals effect has been observed in simple response tasks (e.g., Gondan et al., 2004; Miller, 1982, 1986; Murray et al., 2005), go/no-go tasks (Gondan et al., 2005; Gondan, Vorberg, \& Greenlee, 2007; Miller, 1982, Experiment 3; Teder-Sälejärvi et al., 2005; Teder-Sälejärvi et al., 2002), and choice response tasks (Grice, Canham, \& Boroughs, 1984; Grice, Canham, \& Gwynne, 1984). In simple responses to multisensory stimuli, violations of the race model prediction (Inequality 1) have consistently been reported, ruling out race models for explanation of the redundancy gains observed. Instead, coactivation models (Diederich, 1995; Miller \& Ulrich, 2003; Schwarz, 1989, 1994) have been shown to accurately describe the observed redundancy gains. The integration mechanism of these coactivation models is extremely simple: All models assume a linear, additive superposition of the channel-specific activity. Although reports on superadditive multisensory interactions have dominated the literature on multisensory processes for a long time (e.g., Meredith \& Stein, 1983), most multisensory neurons observed in the superior colliculus integrate their inputs in an additive manner (see, e.g., Stanford, Quessy, \& Stein, 2005). The results of Experiment 1 demonstrate that in simple response tasks, this integration mechanism can greatly improve detection and discrimination.

In bimodal go/no-go tasks, specific coactivation models have not been explicitly tested. To our knowledge, the majority of the studies using go/no-go tasks adopted coactivation mechanisms mainly by rejection of the race model inequality (e.g., Gondan et al., 2004; Gondan et al., 2005; Gondan et al., 2007; Schröger \& Widmann, 1998; Teder-Sälejärvi et al., 2005; Teder-Sälejärvi et al., 2002). This conclusion is incomplete because rejection of Model A does not necessarily imply the validity of Model B. For example, Townsend and Nozawa (1997) have shown that under certain circumstances, serial exhaustive models predict a violation of the race model inequality as well. On the other hand, not all coactivation models predict a violation of Inequality 1 (Ulrich \& Miller, 1997, Table 1).

The two experiments in the present study were designed to test whether the same integration mechanisms can explain the redundant-signals effect in simple and go/no-go responses. Participants had to perform the two tasks using the same auditory-visual stimulus material. We then adjusted task-specific and common diffusion superposition models (Schwarz, 1994) to the mean RTs observed in the two tasks. Since the same stimuli were used for the simple response task and the go/no-go task, we assumed at least a subset of elementary detection processes to be identical in the two tasks. Therefore, in the model fit, the diffusion parameters describing the detection process were constrained to be equal in both tasks.
The evidence criterion and the residual component were allowed to vary as a function of the different task requirements. In Experiment 1, go and no-go stimuli differed in intensity. Here, the fit of an aggregate model for the two tasks was satisfactory, indicating that the participants solved the discrimination task simply by increasing the evidence criterion.

In contrast, in Experiment 2, the fit of a common coactivation model for the two tasks was poor. It was possible, however, to adjust task-specific coactivation models to the mean RTs observed in the two tasks (Table 4), suggesting that coactivation might indeed occur in go/ no-go experiments, but buildup of evidence occurs at a speed different from the simple response task. Indeed, in Participant 1 , the drift rate of the visual process estimated for the simple response task was slightly higher than the visual drift rate estimated for the go/no-go task, whereas the auditory drift rate showed the opposite pattern. In the other 2 participants, we observed "problematic" parameter values for the diffusion variance parameters, suggestive of deterministic evidence accumulation in the auditory channel. At first glance, deterministic processing seems implausible in a biological system. It turned out, however, that the mean RTs predicted by a serial model (Equation 7) can be mimicked by a special case of a deterministic diffusion superposition model with zero diffusion variance parameters (Equation 8). This inclusion enables statistical comparison of the two nested models even though basic mechanisms of the two models differ completely. In Participant 3, it turned out that behavior in the go/no-go task can actually be explained by such a serial self-terminating model. It seems noteworthy that, in this participant, the serial model accounts for the RTs observed both for redundant targets (AV) and for combinations of targets and nontargets $(\mathrm{aV}, \mathrm{Av})$. We hypothesize that response conflicts raised by combinations of targets and nontargets in conditions $\mathrm{Av}$ and $\mathrm{aV}$ (Fournier \& Eriksen, 1990; Grice, Canham, \& Gwynne, 1984) seem to have influenced Participant 3 's processing strategy in the entire experiment, including the redundant go condition (AV).

We stated above that in the redundant-signals task, the serial self-terminating model is mute with respect to unimodal mean RTs. With regard to the classical concept of the bimodal redundancy gain - namely, faster responses for bimodal stimuli than for unimodal stimuli-this might be considered a weakness of the serial model. Although the majority of coactivation models mentioned claim to describe the redundant-signals effect (Diederich, 1995; Miller, 1986, Equation 3; Miller \& Ulrich, 2003; Schwarz, 1989, 1994), it should be underlined that the scope of most of these models is not limited to unimodal and bimodal conditions only (i.e., $\tau=0$ and $\tau=\infty$ ). Rather, these models make specific predictions for arbitrary onset asynchronies, and systematic manipulation of $\tau$ is necessary for testing the majority of them. In other words, the detailed analysis of the mechanisms involved in the integration of information of different sources greatly profits from SOA variation. A broader definition 
of the redundant-signals effect seems, therefore, appropriate that not only includes the special cases $\tau=0$ and $\tau=\infty$, but also covers the function $\tau \rightarrow \mathrm{E}\left[\mathbf{T}_{\mathrm{A}(\tau) \mathrm{V}}\right]$ on a broader range (e.g., Ulrich \& Miller, 1997). ${ }^{3}$ Following this broader definition, the serial self-terminating model describes 11 of the 13 SOA conditions used in the present study.

The fit of the aggregate coactivation model was satisfactory in 2 out of the 3 participants, and it was poor in only 1 out of 3 participants (Table 4 , right columns). Hence, our study does not rule out coactivation in go/ no-go experiments with redundant signals. The results, however, suggest that in bimodal go/no-go discrimination, multisensory integration processes cannot be taken for granted but depend on the exact nature of the stimuli and the task, and they point to the need to consider serial processing when modeling RTs in redundant-signals experiments (Townsend \& Nozawa, 1997).

\section{AUTHOR NOTE}

This research was supported by Grant GO 1855/1-1 to M.G. from the German Research Foundation (DFG). The authors thank Steven Blurton for extensive help during preparation of the manuscript. Correspondence concerning this article should be addressed to M. W. Greenlee, Department of Psychology, University of Regensburg, D-93050 Regensburg, Germany (e-mail: mark.greenlee@psychologie.uni-regensburg.de).

\section{REFERENCES}

Bulmer, M. G. (1979). Principles of statistics. New York: Dover. DE JoNG, R. (1995). The role of preparation in overlapping-task performance. Quarterly Journal of Experimental Psychology, 48A, 2-25.

DiEDERICH, A. (1995). Intersensory facilitation of reaction time: Evaluation of counter and diffusion coactivation models. Journal of Mathematical Psychology, 39, 197-215. doi:10.1006/jmps.1995.1020

Diederich, A., \& Colonius, H. (1987). Intersensory facilitation in the motor component? A reaction time analysis. Psychological Research, 49, 23-29. doi:10.1007/BF00309199

Diederich, A., \& Colonius, H. (1991). A further test of the superposition model for the redundant-signals effect in bimodal detection. Perception \& Psychophysics, 50, 83-86.

Donders, F. C. (1969). On the speed of mental processes. Acta Psychologica, 30, 412-431. (Original work published 1868) doi:10.1016/0001 -6918(69)90065-1

ERIKSEN, C. W. (1988). A source of error in attempts to distinguish coactivation from separate activation in the perception of redundant targets. Perception \& Psychophysics, 44, 191-193.

Eriksen, C. W., \& Hoffman, J. E. (1973). The extent of processing noise elements during selective encoding from visual displays. Perception \& Psychophysics, 14, 155-160.

Fournier, L. R., \& ERIKSEN, C. W. (1990). Coactivation in the perception of redundant targets. Journal of Experimental Psychology: Human Perception \& Performance, 16, 538-550. doi:10.1037/0096 $-1523.16 .3 .538$

Gondan, M. (2009). Testing the race model inequality in redundant stimuli with variable onset asynchrony. Journal of Experimental Psychology: Human Perception \& Performance, 35, 575-579. doi:10.1037/ a0013620

Gondan, M., \& Heckel, A. (2008). Testing the race inequality: A simple correction procedure for fast guesses. Journal of Mathematical Psychology, 52, 322-325. doi:10.1016/j.jmp.2008.08.002

Gondan, M., Lange, K., Rösler, F., \& RöDER, B. (2004). The redundant target effect is affected by modality switch costs. Psychonomic Bulletin \& Review, 11, 307-313.

Gondan, M., Niederhaus, B., Rösler, F., \& RöDer, B. (2005). Multisensory processing in the redundant-target effect: A behavioral and event-related potential study. Perception \& Psychophysics, 67, 713726.
Gondan, M., Vorberg, D., \& Greenlee, M. W. (2007). Modality shift effects mimic multisensory interactions: An event-related potential study. Experimental Brain Research, 182, 199-214. doi:10.1007/ s00221-007-0982-4

Good, I. J. (1973). What are degrees of freedom? American Statistician, 27, 227-228. doi: $10.2307 / 3087407$

Grice, G. R., \& CANHAM, L. (1990). Redundancy phenomena are affected by response requirements. Perception \& Psychophysics, 48, 209-213.

Grice, G. R., Canham, L., \& Boroughs, J. M. (1984). Combination rule for redundant information in reaction time tasks with divided attention. Perception \& Psychophysics, 35, 451-463.

Grice, G. R., Canham, L., \& GWynne, J. W. (1984). Absence of a redundant-signals effect in a reaction time task with divided attention. Perception \& Psychophysics, 36, 565-570.

Grice, G. R., \& ReED, J. M. (1992). What makes targets redundant? Perception \& Psychophysics, 51, 437-442.

LucE, R. D. (1986). Response times: Their role in inferring mental organization. New York: Oxford University Press.

LURIA, R., \& MEIRAN, N. (2005). Increased control demand results in serial processing. Evidence from dual-task performance. Psychological Science, 16, 833-840. doi:10.1111/j.1467-9280.2005.01622.x

Meredith, M. A., \& Stein, B. E. (1983). Interactions among converging sensory inputs in the superior colliculus. Science, 221, 389-391. doi:10.1126/science. 6867718

Miller, J. (1982). Divided attention: Evidence for coactivation with redundant signals. Cognitive Psychology, 14, 247-279. doi:10.1016/ 0010-0285(82)90010-X

Miller, J. (1986). Timecourse of coactivation in bimodal divided attention. Perception \& Psychophysics, 40, 331-343.

Miller, J., \& LOPES, A. (1991). Bias produced by fast guessing in distribution-based tests of race models. Perception \& Psychophysics, 50, 584-590.

Miller, J., \& UlRich, R. (2003). Simple reaction time and statistical facilitation: A parallel grains model. Cognitive Psychology, 46, 101151. doi:10.1016/S0010-0285(02)00517-0

Murray, M. M., Molholm, S., Michel, C. M., Heslenfeld, D. J., RitTeR, W., JaVITT, D. C., ET AL. (2005). Grabbing your ear: Rapid auditory-somatosensory multisensory interactions in low-level sensory cortices are not constrained by stimulus alignment. Cerebral Cortex, 15, 963-974. doi:10.1093/cercor/bhh197

RAAB, D. H. (1962). Statistical facilitation of simple reaction times. Transactions of the New York Academy of Sciences, 24, 574-590.

R Development Core Team (2009). R: A language and environment for statistical computing. Vienna: R Foundation for Statistical Computing. Available at www.r-project.org/.

Schröger, E., \& Widmann, A. (1998). Speeded responses to audiovisual signal changes result from bimodal integration. Psychophysiology, 35, 755-759. doi:10.1017/S0048577298980714

SCHWARz, W. (1989). A new model to explain the redundant-signals effect. Perception \& Psychophysics, 46, 498-500.

Schwarz, W. (1994). Diffusion, superposition, and the redundant targets effect. Journal of Mathematical Psychology, 38, 504-520. doi:10.1006/jmps.1994.1036

Schwarz, W. (2006). On the relationship between the redundant signals effect and temporal order judgments: Parametric data and a new model. Journal of Experimental Psychology: Human Perception \& Performance, 32, 558-573. doi:10.1037/0096-1523.32.3.558

Schweickert, R., Giorgini, M., \& Dzhafarov, E. (2000). Selective influence and response time cumulative distribution functions in serial-parallel task networks. Journal of Mathematical Psychology, 44, 504-535. doi:10.1006/jmps. 1999.1268

Stanford, T. R., Quessy, S., \& Stein, B. E. (2005). Evaluating the operations underlying multisensory integration in the cat superior colliculus. Journal of Neuroscience, 25, 6499-6508. doi:10.1523/ JNEUROSCI.5095-04.2005

Teder-Sälejärvi, W. A., Di Russo, F., McDonald, J. J., \& Hillyyard, S. A. (2005). Effects of spatial congruity on audio-visual multimodal integration. Journal of Cognitive Neuroscience, 17, 1396-1409. doi:10.1162/0898929054985383

Teder-Sälejärvi, W. A., McDonald, J. J., Di Russo, F., \& Hillyard, S. A. (2002). An analysis of audio-visual crossmodal integration by 
means of event-related potential (ERP) recordings. Cognitive Brain Research, 14, 106-114. doi:10.1016/S0926-6410(02)00065-4

Tolhurst, D. J., Movshon, J. A., \& Dean, A. F. (1983). The statistical reliability of signals in single neurons in cat and monkey visual cortex. Vision Research, 23, 775-785. doi:10.1016/0042-6989(83)90200-6

Townsend, J. T., \& Ashby, F. G. (1983). Stochastic modeling of elementary psychological processes. Cambridge: Cambridge University Press.

Townsend, J. T., \& Nozawa, G. (1995). Spatio-temporal properties of elementary perception: An investigation of parallel, serial, and coactive theories. Journal of Mathematical Psychology, 39, 321-359. doi:10.1006/jmps.1995.1033

Townsend, J. T., \& Nozawa, G. (1997). Serial exhaustive models can violate the race model inequality: Implications for architecture and capacity. Psychological Review, 104, 595-602. doi:10.1037/0033 $-295 X .104 .3 .595$

UlRiCH, R., \& MilleR, J. (1997). Tests of race models for reaction time in experiments with asynchronous redundant signals. Journal of Mathematical Psychology, 41, 367-381. doi:10.1006/jmps.1997.1181

WELCH, R. B., \& WARREN, D. H. (1986). Intersensory interactions. In K. R. Boff, L. Kaufman, \& J. P. Thomas (Eds.), Handbook of perception and human performance (pp. 25/1-25/36). New York: Wiley.

\section{NOTES}

1. Stated differently, the mixture model predicts a zero mean interaction $\mathrm{E}\left[\mathbf{T}_{\mathrm{AV}}\right]+\mathrm{E}\left[\mathbf{T}_{\mathrm{AV}}\right]+\tau=\mathrm{E}\left[\mathbf{T}_{\mathrm{A}(\tau) \mathrm{V}}\right]+\mathrm{E}\left[\mathbf{T}_{\mathrm{V}(\tau) \mathrm{A}}\right]$, a result similar to that observed by Townsend and Nozawa (1995, p. 329).

2 . The full description of the relationship between SOA and the probability $\pi$ for processing the visual modality first requires further assumptions, differentially accounting for the large SOA conditions in Equations 7-9, on the one hand, and Equation 10, on the other hand. Thus, for pragmatic reasons, the system was assumed to be rigid with respect to onset asynchrony, with the probability $\pi$ being constant over the entire range of finite SOAs used in the present study. The goodness of fit estimated by such a rigid model can serve as a pessimistic bound of the "true" goodness of fit of a more flexible serial self-terminating model.

3. Or, more generally, $\tau \rightarrow f(\tau)$, with $f$ denoting mean, median, and so forth.

\section{SUPPLEMENTAL MATERIALS}

Detailed RTs from the experiments discussed in this article may be downloaded from http://app.psychonomic-journals.org/content/ supplemental.

\section{APPENDIXA \\ Violation of the Race Model Inequality Predicted by the Superposition Model}

The diffusion superposition model describes processing of a stimulus by a time-homogeneous diffusion process with drift $\mu>0$ and variance $\sigma^{2}>0$ (Wiener process). Detection occurs if an absorbing barrier $c>0$ (criterion) is passed for the first time. These first-passage times follow an inverse Gaussian distribution with distribution $W(t)=\mathrm{P}(\mathbf{D} \leq t)$ and density

$$
w(t)=c / \sqrt{ }\left(2 \pi \sigma^{2} t^{3}\right) \times \exp \left[-(c-\mu t)^{2} /\left(2 \sigma^{2} t\right)\right] .
$$

In redundant stimuli (e.g., AV), the two processes superimpose to an aggregate process with drift $\mu_{\mathrm{AV}}=\mu_{\mathrm{A}}+\mu_{\mathrm{V}}$ and variance $\sigma_{\mathrm{AV}}^{2}=\sigma_{\mathrm{A}}^{2}+\sigma_{\mathrm{V}}^{2}+2 \rho_{\mathrm{AV}} \sigma_{\mathrm{A}} \sigma_{\mathrm{V}}$, with $\rho_{\mathrm{AV}}$ denoting the correlation of the two processes (Schwarz, 1994, p. 507). In Schwarz (1994) and in the present study, the parameter $\rho_{\mathrm{AV}}$ is assumed to be zero.

Here, we demonstrate that under this assumption, the diffusion superposition model predicts a violation of the race model inequality,

$$
W_{\mathrm{AV}}(t)>W_{\mathrm{A}}(t)+W_{\mathrm{V}}(t), \text { for small } t,
$$

with $W(t)$ denoting the inverse Gaussian distribution functions $W(t)={ }_{0}{ }^{t} w(x) \mathrm{d} x$. To this end, it is sufficient to demonstrate $w_{\mathrm{AV}}(t)>w_{\mathrm{A}}(t)+w_{\mathrm{V}}(t)$, for small $t$. For small $t$, however, the exponential in Equation A1 decomposes into three factors: $-c^{2} /\left(2 \sigma^{2} t\right)$ is the relevant term, whereas $2 \mu t /\left(2 \sigma^{2} t\right)$ reduces to a positive constant and $-(\mu t)^{2} /\left(2 \sigma^{2} t\right)$ vanishes. The problem, therefore, reduces to showing that $s_{\mathrm{AV}}(t)>s_{\mathrm{A}}(t)+s_{\mathrm{V}}(t)$ or, equivalently, $s_{\mathrm{AV}}(t) /\left[s_{\mathrm{A}}(t)+s_{\mathrm{V}}(t)\right]>1$, for small $t$, with

$$
\begin{aligned}
& s_{\mathrm{AV}}(t)=1 / \sqrt{ }\left(\sigma_{\mathrm{AV}}^{2} t^{3}\right) \times \exp \left[-c^{2} /\left(2 \sigma_{\mathrm{AV}}^{2} t\right)\right], \\
& s_{\mathrm{A}}(t)=1 / \sqrt{ }\left(\sigma_{\mathrm{A}}^{2} t^{3}\right) \times \exp \left[-c^{2} /\left(2 \sigma_{\mathrm{A}}^{2} t\right)\right], \\
& s_{\mathrm{V}}(t)=1 / \sqrt{ }\left(\sigma_{\mathrm{V}}^{2} t^{3}\right) \times \exp \left[-c^{2} /\left(2 \sigma_{\mathrm{V}}^{2} t\right)\right] .
\end{aligned}
$$

Without loss of generality, we assume $\sigma_{\mathrm{A}} \leq \sigma_{\mathrm{V}}$ and note that $s_{\mathrm{A}}(t) \leq 1 / \sqrt{ }\left(\sigma_{\mathrm{A}}^{2} t^{3}\right) \times \exp \left[-c^{2} /\left(2 \sigma_{\mathrm{V}}^{2} t\right)\right]$, as well as $s_{\mathrm{V}}(t) \leq 1 / \sqrt{ }\left(\sigma_{\mathrm{A}}^{2} t^{3}\right) \times \exp \left[-c^{2} /\left(2 \sigma_{\mathrm{V}}^{2} t\right)\right]$, such that $s_{\mathrm{A}}(t)+s_{\mathrm{V}}(t) \leq s_{\max }(t)$, with

$$
s_{\max }(t)=2 / \sqrt{ }\left(\sigma_{\mathrm{A}}^{2} t^{3}\right) \times \exp \left[-c^{2} /\left(2 \sigma_{\mathrm{V}}^{2} t\right)\right] .
$$

It is now easily seen that

$$
s_{\mathrm{AV}}(t) / s_{\max }(t)=1 / 2 \sigma_{\mathrm{A}} / \sigma_{\mathrm{AV}} \times \exp \left[-c^{2} /\left(2 \sigma_{\mathrm{AV}}^{2} t\right)\right] \times \exp \left[-c^{2} /\left(2 \sigma_{\mathrm{V}}^{2} t\right)\right]
$$

is greater than 1 for small $t$, as long as $\sigma_{\mathrm{AV}}^{2}>\sigma_{\mathrm{V}}^{2}$. This is established if $\rho_{\mathrm{AV}}$ is not too negative, which is given by the assumption $\rho_{\mathrm{AV}}=0$. 


\section{APPENDIX B}

\section{Serial Self-Terminating Model}

Townsend and Nozawa (1997) have demonstrated that serial exhaustive processing might cause violations of the race model inequality. Here we will show that serial self-terminating processing of redundant signals is compatible with the race model inequality. As was mentioned in the main text, in redundant signals, both stimulus components are targets, such that the serial self-terminating model reduces to a mixture model in which only one stimulus (attended stimulus) is processed in each trial. We assume context independence; that is, the component-specific processing time distributions and the probability $\pi$ for processing the visual component first are invariant over the SOA range, including unimodal stimuli. For a given single modality stimulus, the probability for responding within $t$ corresponds to the weighted sum of the probabilities conditional on whether the modality is attended or not,

$$
\begin{aligned}
& \mathrm{P}\left\{\mathbf{T}_{\mathrm{A}} \leq t\right\}=(1-\pi) \mathrm{P}\left\{\mathbf{T}_{\mathrm{A}} \leq t \mid \mathrm{A} \text { attended }\right\}+\pi \mathrm{P}\left\{\mathbf{T}_{\mathrm{A}} \leq t \mid \mathrm{V} \text { attended }\right\} \\
& \mathrm{P}\left\{\mathbf{T}_{\mathrm{V}} \leq t\right\}=(1-\pi) \mathrm{P}\left\{\mathbf{T}_{\mathrm{V}} \leq t \mid \mathrm{A} \text { attended }\right\}+\pi \mathrm{P}\left\{\mathbf{T}_{\mathrm{V}} \leq t \mid \mathrm{V} \text { attended }\right\} .
\end{aligned}
$$

If, in a given unimodal trial, the participant attends to the wrong channel, we assume that the participant will wait indefinitely for the attended stimulus to appear, $\mathrm{P}\left\{\mathbf{T}_{\mathrm{A}} \leq t \mid \mathrm{V}\right.$ attended $\}=\mathrm{P}\left\{\mathbf{T}_{\mathrm{V}} \leq t \mid \mathrm{A}\right.$ attended $\}=0$. Under this assumption, the distributions $\mathrm{P}\left\{\mathbf{T}_{\mathrm{A}} \leq t\right\}$ and $\mathrm{P}\left\{\mathbf{T}_{\mathrm{V}} \leq t\right\}$ are incomplete (i.e., they range between 0 and less than 1), because the participant omits the responses to the unattended stimuli (this assumption will be relaxed below).

For bimodal stimuli, the processing time distribution corresponds to the mixture of the component-specific processing time distributions:

$$
\mathrm{P}\left\{\mathbf{T}_{\mathrm{AV}} \leq t\right\}=(1-\pi) \mathrm{P}\left\{\mathbf{T}_{\mathrm{A}} \leq t \mid \mathrm{A} \text { attended }\right\}+\pi \mathrm{P}\left\{\mathbf{T}_{\mathrm{V}} \leq t \mid \mathrm{V} \text { attended }\right\} .
$$

It is immediately evident from Equations $\mathrm{B} 1$ and $\mathrm{B} 2$ that the mixture model predicts the race model inequality to hold exactly- that is, $\mathrm{P}\left\{\mathbf{T}_{\mathrm{AV}} \leq t\right\}=\mathrm{P}\left\{\mathbf{T}_{\mathrm{A}} \leq t\right\}+\mathrm{P}\left\{\mathbf{T}_{\mathrm{V}} \leq t\right\}$, for finite $t$.

If, in unimodal trials, the participant does not wait indefinitely for a stimulus in the attended modality but, after some delay, switches to the other modality, the observed proportions $\mathrm{P}\left\{\mathbf{T}_{\mathrm{A}} \leq t\right\}$ and $\mathrm{P}\left\{\mathbf{T}_{\mathrm{V}} \leq t\right\}$ will be increased, which is still consistent with the race model inequality. 